Pure and Applied Mathematics Quarterly

Volume 8, Number 1

(Special Issue: In honor of

F. Thomas Farrell and Lowell E. Jones, Part 1 of 2)

$133-173,2012$

\title{
Factoring the Becker-Gottlieb Transfer Through the Trace Map
}

\author{
Wojciech Dorabiała and Mark W. Johnson
}

\begin{abstract}
In 1998, Becker and Schultz [6] published axioms characterizing the Becker-Gottlieb transfer $\tau_{B G}(p): \Sigma^{\infty}\left(B_{+}\right) \rightarrow \Sigma^{\infty}\left(E_{+}\right)$for certain types of fibrations $p: E \rightarrow B$. We verify these axioms for the composite of the algebraic K-theory transfer $\tau_{\mathrm{K}}(p): \Sigma^{\infty}\left(B_{+}\right) \rightarrow \mathrm{A}(E)$ of any perfect fibration $p$ followed by the evaluation (at the unit) from the free loop space $\Lambda$ of the Bökstedt trace map tr : $\mathrm{A}(E) \rightarrow \Sigma^{\infty}\left(\Lambda E_{+}\right) \rightarrow \Sigma^{\infty}\left(E_{+}\right)$. As a consequence, for $p$ any compact ANR fibration with finite $\mathrm{CW}$ base (those considered by Becker-Shultz), $\tau_{B G}(p) \simeq \operatorname{tr} \tau_{\mathrm{K}}(p)$.
\end{abstract}

Keywords: transfer, trace, algebraic K-theory of spaces, topological Hochschild homology.

\section{INTRODUCTION}

In many situations in mathematics, it is important to understand the collection of automorphisms of commonly occurring objects. Thus, much work in the theory of smooth manifolds has focused on understanding $\operatorname{Dif} f(M)$, the space of self-diffeomorphisms of a compact, smooth manifold $M$. Trying to use the tools of homotopy theory leads one to consider the space of (smooth) paths in $\operatorname{Diff}(M)$ starting at the identity, which is the isotopy space $I s o(M)$. In 1970, J. Cerf [12] introduced the (smooth) pseudoisotopy (or concordance) space $P(M)$, consisting

Received October 17, 2007.

1991, Mathematics Subject Classification. Primary: 19D10; Secondary: 18F25, 19Exx, $55 \mathrm{R} 70$. 
of all self-diffeomorphisms of the cylinder $M \times I$ which leave $M \times 0 \cup \partial M \times I$ fixed pointwise. Notice, by adjointness, an isotopy can also be viewed as a selfdiffeomorphism of $M \times I$ which leaves $M \times 0$ fixed and preserves the second coordinate, so $I s o(M) \subset P(M)$. In fact, whenever $P(M)$ is path connected, the more convenient tools of pseudoisotopy are sufficient to study the path components of Diff $(M)$, and Cerf showed that $M$ without boundary, simply connected, and dimension at most 6 implies $P(M)$ is path connected.

In order to have more room for homotopies, one next passes to the stable smooth pseudoisotopy space, $\mathcal{P}(M)$, given by the homotopy colimit of the tower of "suspension maps"

$$
P(M) \rightarrow P(M \times I) \rightarrow P\left(M \times I^{2}\right) \rightarrow \ldots .
$$

Igusa's Stability Theorem [21] shows the induced stablization map $P(M) \rightarrow$ $\mathcal{P}(M)$ induces an isomorphism for homotopy groups below roughly one third the dimension of $M$. A delooping of $\mathcal{P}(M)$ is given by the (smooth) stable h-cobordism space of $M$, which is built from the space of h-cobordisms via "suspension maps" as well. A second (or double) delooping of $\mathcal{P}(M)$, by work of Waldhausen [32], is given by the (smooth) Whitehead space $W h^{\text {diff }}(M)$.

Using a version of the trace map and topological Hochschild homology, Waldhausen also showed [30] that there is a natural splitting of infinite loop spaces

$$
\mathrm{A}(M) \simeq \Sigma^{\infty}\left(M_{+}\right) \times W h^{\operatorname{diff}}(M),
$$

where $\mathrm{A}(M)$ is his algebraic K-theory of spaces, which we refer to as A-theory. Combined with Igusa's Stability Theorem, this allows one to recover information about the homotopy groups of $P(M)$ (hence indirectly about $\operatorname{Dif} f(M)$ ) from information about $\mathrm{A}(M)$ (and the stable homotopy of the manifold, $\Sigma^{\infty}\left(M_{+}\right)$).

Another important tool in the study of pseudoisotopy is a geometric transfer map for pseudoisotopy spaces. Given $p: N \rightarrow M$ a smooth fiber bundle with compact fibers, this is a map $p^{*}: \mathcal{P}(M) \rightarrow \mathcal{P}(N)$, which also passes to the second delooping $p^{*}: W h^{\operatorname{diff}}(M) \rightarrow W h^{\operatorname{diff}}(N)$. Burghelea [11] used these geometric transfer maps for (stable smooth) pseudoisotopy spaces to study the homotopy groups of $\operatorname{Diff}(M)$. Under the same condition, there are also abstract transfer maps in A-theory, $\tau_{\mathrm{A}}(p): \mathrm{A}(M) \rightarrow \mathrm{A}(N)$, which Lück [25], [24] verified were compatible with the geometric transfers on $W h^{\operatorname{diff}}(M)$ via the splitting above, 
at least for $\pi_{0}$ and $\pi_{1}$. Thus, one natural question is to understand the restriction of $\tau_{\mathrm{A}}(p)$ to the stable homotopy factor.

Recall that Waldhausen's splitting result has a weaker formulation, saying the composite of the trace and the assembly map

$$
\Sigma^{\infty}\left(X_{+}\right) \stackrel{\alpha}{\longrightarrow} \mathrm{A}(X) \stackrel{\operatorname{tr}}{\longrightarrow} \Sigma^{\infty}\left(X_{+}\right)
$$

is naturally homotopy equivalent to the identity. Then, with $\tau_{\mathrm{K}}(p)=\tau_{\mathrm{A}}(p) \alpha$ : $\Sigma^{\infty}\left(X_{+}\right) \rightarrow \mathrm{A}(E)$ denoting the Algebraic K-theory transfer, identifying the portion of the A-theory transfer visible using stable homotopy, $\left.\operatorname{tr} \tau_{\mathrm{A}}(p)\right|_{\Sigma^{\infty}\left(M_{+}\right)}$, is equivalent to determining $\operatorname{tr} \tau_{\mathrm{A}}(p) \alpha=\operatorname{tr} \tau_{\mathrm{K}}(p)$. As a consequence of work of Dwyer, Weiss, and Williams [17], one sees $\left.\operatorname{tr} \tau_{\mathrm{A}}(p)\right|_{\Sigma^{\infty}\left(M_{+}\right)}$is the BeckerGottlieb(-Dold) transfer for $p$ a smooth fiber bundle. The primary goal of the current article is to extend this identification for a more general class of fibrations, by exploiting the axiomatic characterization of the Becker-Gottlieb transfer given by Becker and Schultz [6] and stated in our language in Def. 2.15 and Thm 2.16.

Note that in the special case of a disk, $A\left(D^{n}\right)=\Sigma^{\infty}\left(S^{0}\right) \times W h^{\operatorname{diff}}\left(D^{n}\right)$ is related to the pseudoisotopy (and $h$-cobordism) space of a disc (and the stable homotopy groups of spheres). In this (contractible) case, Douglas [15] showed $\operatorname{tr} \tau_{\mathrm{K}}(p)$ is the Becker-Gottlieb transfer for $p$ any compact ENR-fibration over the disk as part of a more general result. The main result of [15] shows that for a fibration $p: E \rightarrow X$ with compact fibers making the total space an ENR over the base, the following commutes (up to weak homotopy)

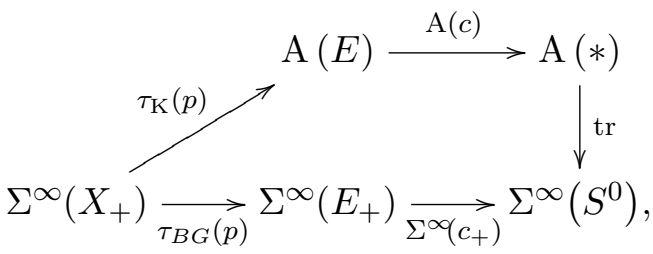

where $c: E \rightarrow *$ is the collapse map.

The main result here is the following: 
Theorem 2.17. If $p: E \rightarrow X$ is a compact ANR fibration with finite $C W$ base, then the diagram

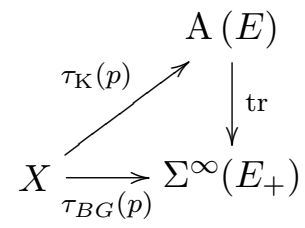

commutes in the homotopy category of spectra.

The first author has been investigating, along with Badzioch [2] as well as both authors with Williams [3], applications of this theorem to computations of higher Reidemeister torsion (after Dwyer, Weiss, and Williams [17]).

As mentioned above, the theorem is known for smooth fiber bundles, as a consequence of work of Dwyer,Weiss, and Williams [17], where it is shown that for a smooth fiber bundle $p: E \rightarrow X$, the diagram

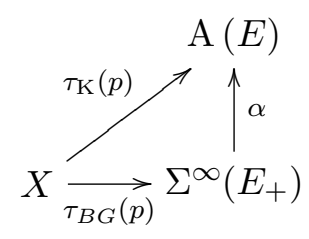

commutes up to homotopy. If one follows each map to $\mathrm{A}(E)$ with the trace map, $\mathrm{A}(E) \rightarrow \Sigma^{\infty}\left(E_{+}\right)$, Waldhausen's result in [32] that the trace of the assembly map is homotopic to the identity implies

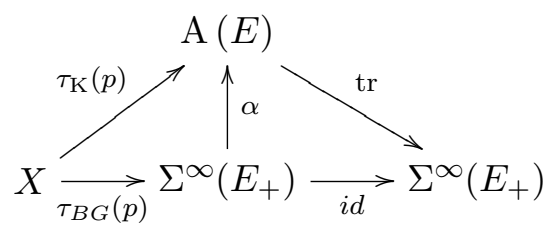

commutes up to homotopy (still in the smooth case) and the lower horizontal is also $\tau_{B G}(p)$.

Our method of proof for Theorem 2.17 will be to verify that the axioms for characterizing the Becker-Gottlieb transfer given by Becker and Schultz [6] are satisfied by the composite of the trace with the algebraic K-theory transfer. In fact, we will verify that each of these axioms holds (including the stronger version of additivity) for perfect fibrations, that is Hurewicz fibrations whose fibers are retracts up to homotopy of finite $\mathrm{CW}$-cmplexes. Hence, if it could be shown that 
these same axioms characterize the Becker-Gottlieb transfer for any larger class of perfect fibrations, (consider the preprint of Klein and Williams [23]) then our result would also extend immediately.

In order to apply the axioms of Becker and Schultz [6], we must extend to a relative version each of the relevant natural maps, and verify compatibility of these extensions with the natural relative external products along with a relative additivity result. Since we rely upon maps induced on homotopy cofiber constructions for our relative maps, we cannot simply work in the homotopy category. We must instead work with weak maps (see subsection 2.5 below) and study their multiplicative properties carefully. Fortunately, all of the weak maps of importance to us consist of natural pieces, so a CW pair $(X, Y)$ induces a string of commutative squares (not just homotopy commutative squares, a key detail for us). As a consequence, there is an induced map on the homotopy cofibers associated to the inclusion at each intermediate stage of the original weak map, and we concatenate these into a relative weak map (see Lemma 2.6 and Rem. 2.7). Of course, this introduces substantial technical complexities, particularly for tracking multiplicative properties, which we address in the earlier sections.

Section 2 is devoted to background material familiar to the experts in this area, along with our definitions for the (relative) A-theory transfer (following Williams [36]), assembly (following Weiss and Williams [35]) and trace maps (following Bökstedt [7]). The section ends with a statement of the axioms and main result of Becker and Schultz (Def. 2.15 and Thm 2.16) in the current language, as well as our primary result (Thm 2.17). Section 3 is focused on the rather involved details of the compatibility of the earlier constructions with external pairings, and includes the verification of the multiplicativity (Prop. 3.8) of our candidate transfer. The short section 4 is then devoted to verifying the normalization (Prop. 4.2) and naturality (Prop. 4.1) properties for our candidate transfer, relying mainly on details presented earlier.

Section 5 is dedicated to verifying the strong additivity axiom, which is more complicated. The key underlying result is the Transfer Additivity Theorem of the first author [13] and [2]. Unfortunately, that result does not include a naturality statement for the relevant homotopy, hence it need not extend to an induced map on homotopy cofibers. This requires us to work with a less well-known model for the algebraic K-theory transfer, following Dwyer, Weiss, and Williams [17], which 
is defined in terms of parametrized A-theory, and closer to that used by Douglas [15].

\section{NOtATION AND BACKGROUND}

This section will introduce material already familiar to experts in this area, along with some notation. Included are various definitions for retractive spaces, A-theory, the bivariant A-theory introduced by Williams, and a relative version of his view of the A-theory transfer, which was inspired by unpublished work of Waldhausen. This is followed by a careful discussion of weak natural transformations, along with the relative assembly and trace maps. The section ends with a restatement of the axioms of Becker and Schultz, along with their main theorem, and the statement of our main result.

2.1. Classes of Fibrations. First, there are several different classes of (Hurewicz) fibrations (usually denoted $p: E \rightarrow X$ ) considered in our references. We would like to work with the class of perfect fibrations following Williams [36], that is, fibrations whose fibers are retracts up to homotopy of finite CW complexes. This class will contain all of the others, so our results will apply to each of the other classes as well. We will also assume the base space $X$ has the homotopy type of a CW complex. Among other things, this means $\Sigma^{\infty}\left(X_{+}\right)$will have the homotopy type of a CW spectrum, so the Hurewicz Theorem will imply any weak homotopy equivalence between spectra of this type will actually be a homotopy equivalence. Thus, we avoid the necessity of working with weak homotopy equivalences in many cases.

Given a CW inclusion $i: Y \rightarrow X$, a perfect fibration of pairs $p=\left(p_{X}, p_{Y}\right)$ : $\left(E_{X}, E_{Y}\right) \rightarrow(X, Y)$, consists of a perfect fibration $p_{X}$ and its restriction to the subspace $Y$ (which is then also a perfect fibration).

Becker and Schultz [6] work with compact ANR fibrations when establishing their axioms characterizing the Becker-Gottlieb transfer $\tau_{B G}(p): X \rightarrow \Sigma^{\infty}\left(E_{+}\right)$. That is, they consider fiber bundles $p: E \rightarrow X$ with $X$ a finite CW complex and fibers finite dimensional compact ANRs (Absolute Neighborhood Retracts). As they emphasize, an important consequence is that $E$ then also has the homotopy type of a finite $\mathrm{CW}$ complex. 
A different class of fibrations, considered by Douglas [15], are the compact ENR fibrations, that is fibrations with compact fibers that make the total space an ENR (Euclidean Neighborhood Retract) over the base.

2.2. Retractive Spaces. A retractive space over $X$ consists of a space $Z$, together with maps $i: X \rightarrow Z$ and $r: Z \rightarrow X$ such that $r \circ i$ is the identity on $X$. Clearly this becomes a category $\operatorname{Ret}(X)$ if as morphisms from $(Z, i, r)$ to $\left(Z^{\prime}, i^{\prime}, r^{\prime}\right)$ one takes all (continuous) maps $f: Z \rightarrow Z^{\prime}$ with $f \circ i=i^{\prime}$ and $r=r^{\prime} \circ f$. It is common to leave the choice of $i$ and $r$ out of the notation whenever possible without confusion.

Given $M \in \operatorname{Ret}(X)$ and $N \in \operatorname{Ret}(W)$, their external coproduct is the element of Ret $(X \times W)$ defined by the pushout diagram (with both maps coming from the structural inclusions)

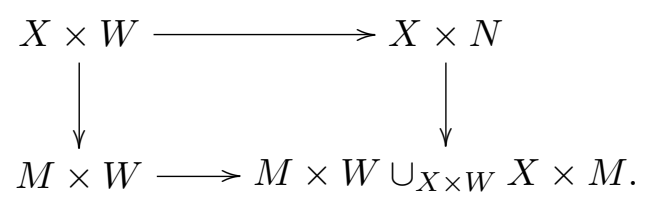

We will use $M_{X} \vee_{W} N$ as a short form of $M \times W \cup_{X \times W} X \times M$.

Similarly, the external (smash) product is the element of $\operatorname{Ret}(X \times W)$ given by the pushout diagram

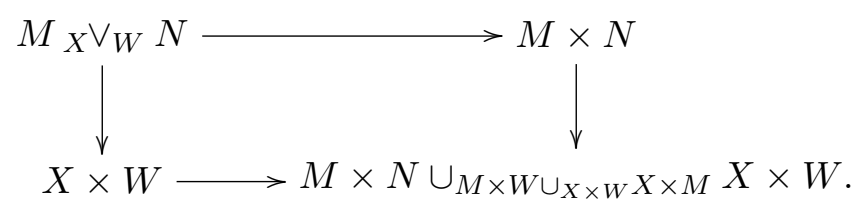

We will use $M_{X} \wedge_{W} N$ as a short form of $M \times N \cup_{M \times W \cup_{X \times W} X \times M} X \times W$.

A retractive space $Y$ over $X$ is homotopy finite if there is a morphism of retractive spaces $W \rightarrow Y$ which is an underlying homotopy equivalence, such that the structure map $X \rightarrow W$ is the inclusion of a finite relative CW complex. One calls $Z$ (homotopy) finitely dominated over $X$ if there exists a composition in the category of retractive spaces $Z^{\prime} \rightarrow Y \rightarrow Z$ whose underlying composite is a homotopy equivalence of spaces, such that $Y$ is homotopy finite. We will use $\operatorname{Ret}^{f d}(X)$ to indicate the full subcategory of retractive spaces over $X$ with objects the finitely dominated retractive spaces. This is a Waldhausen category (that is, 
a category with cofibrations and weak equivalences in Waldhausen's terminology [33]) where the cofibrations are the closed embeddings satisfying the homotopy extension property (the closed cofibrations of [29]) and weak equivalences are the homotopy equivalences (see [17, II.6.1] for details). Thus, we can apply Waldhausen's K-theory functor, which yields the spectrum $\mathrm{A}(X)$ in this case.

2.3. Bivariant A-theory. More generally, given $p: E \rightarrow X$, one calls a retractive space over $E$ fiberwise finitely dominated over $p$ if each homotopy fiber of $p \circ r$ at $x \in X$ is finitely dominated over the fiber of $p$ at $x, F_{x}$. We will use $\operatorname{Ret}^{f d}(p)$ to indicate the full subcategory of retractive spaces over $E$ with objects that are fiberwise finitely dominated over $p$. This is a sub-Waldhausen category of $\operatorname{Ret}^{f d}(E)$, so we can again apply Waldhausen's K-theory functor, which yields the spectrum $\mathrm{A}(p)$ in this case, the Bivariant A-theory of Williams [36]. One particularly important thing to notice here is that $p: E \rightarrow X$ a perfect fibration implies $E \amalg E \rightarrow E \rightarrow X$ is fiberwise finitely dominated over $p$. This yields a point $\chi(p)$ in $\mathrm{A}(p)$ which Williams [36] uses to give a particularly useful description of the transfer map in the algebraic K-theory of spaces, also exploited by the first author alone [13], with Badzioch [2], and with the second author [14]. We will later refer to this point in the A-theory spectrum as the homotopy parametrized Euler characteristic of the fibration.

A CW-inclusion $i: Y \rightarrow X$ induces an exact functor $i_{*}: \operatorname{Ret}^{f d}(Y) \rightarrow$ $\operatorname{Ret}^{f d}(X)$ and we use $i_{*} \operatorname{Ret}^{f d}(Y)$ to denote its replete image, that is the full subcategory of $\operatorname{Ret}^{f d}(X)$ consisting of objects isomorphic to some $i_{*}(W)$. If we give $i_{*} \operatorname{Ret}^{f d}(Y)$ the Waldhausen structure it inherits from $\operatorname{Ret}^{f d}(X)$, then $i_{*}$ even becomes an equivalence of Waldhausen categories $\operatorname{Ret}^{f d}(Y) \rightarrow i_{*} \operatorname{Ret}^{f d}(Y)$, since $i$ is a CW-inclusion by assumption. Also, recall that a "quotient" of retractive spaces over $X$ is defined by the pushout

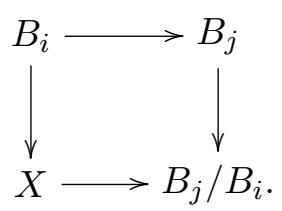

2.4. Constructing the (Relative) A-theory transfer. Given a perfect fibration $p: E \rightarrow X$, it follows from [1, Lemma 3.1] that finite domination is preserved under pullback over the fibration. As a consequence, one has a pullback functor 
$\operatorname{Ret}^{f d}(X) \rightarrow \operatorname{Ret}^{f d}(E)$ which is exact, as is readily verified using the following Lemma due to Lück. An earlier formulation of this result is available at the end of $[29]$.

Lemma 2.1 (Lemma 1.26 of Lück [26]). Suppose

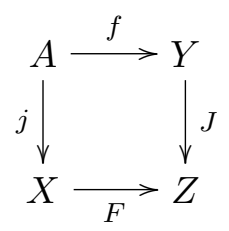

is a pushout diagram while $j$ is a (closed) cofibration. Given a fibration $p: E \rightarrow$ $Z$, the pullback construction yields a pushout diagram

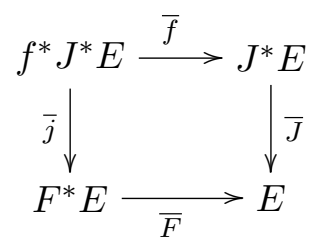

with $\bar{j}$ also a (closed) cofibration.

As an exact functor, this pullback construction induces a map on A-theory, known as the A-theory transfer. We will generally use notation like $p_{X}^{*}$ (or even $p^{*}$ when no confusion will result) for such transfers. Given a perfect fibration of pairs (see subsection 2.1), there is a commutative diagram of Waldhausen categories and exact functors

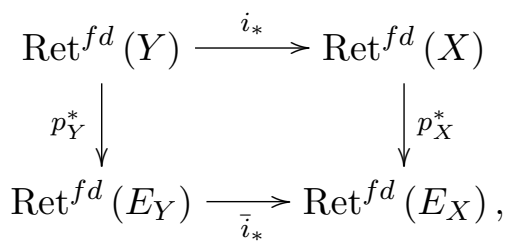

since $p_{Y}$ is simply the restriction of $p_{X}$ (and the singleton is assumed to be unique). As a consequence, the induced diagram

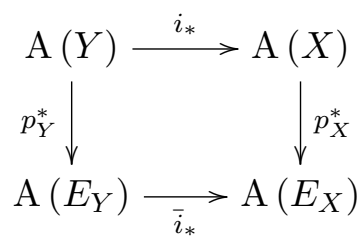


commutes as well.

Let hocofib indicate the Bousfield-Kan [10] model for the homotopy cofiber of spectra.

Definition 2.2. Given a functor $G$ from spaces to spectra, define its extension to $C W$ pairs by

$$
G(X, Y)=\operatorname{hocofib}(G(i): G(Y) \rightarrow G(X))
$$

for $i: Y \rightarrow X$ a CW inclusion.

Then we have our relative A-theory transfer by functoriality of hocofib.

Lemma 2.3. For any perfect fibration of pairs $\left(p_{X}, p_{Y}\right):\left(E_{X}, E_{Y}\right) \rightarrow(X, Y)$, there is an induced relative $\mathrm{A}$-theory transfer $\tau_{\mathrm{A}}\left(p_{X}, p_{Y}\right): \mathrm{A}(X, Y) \rightarrow \mathrm{A}\left(E_{X}, E_{Y}\right)$. Furthermore, given a fiberwise homotopy equivalence $f: E_{W} \rightarrow E_{X}$ making the diagram

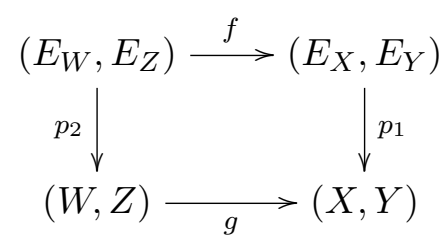

commute, the naturality diagram

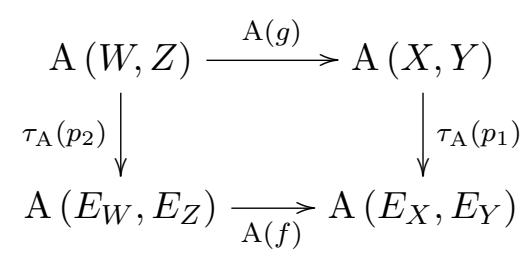

commutes up to homotopy.

Proof. The existence follows from functoriality of hocofib and the commutative diagram above Def. 2.2. Now we decompose the naturality result into three special cases, by first factoring $g$ as a cofibration $k$ followed by a homotopy equivalence $q$. Then define $p_{3}$ to be the pullback of $p_{1}$ over $q$, and $p_{4}$ the pullback 
of $p_{3}$ over $k$. This splits the original diagram into three pieces,

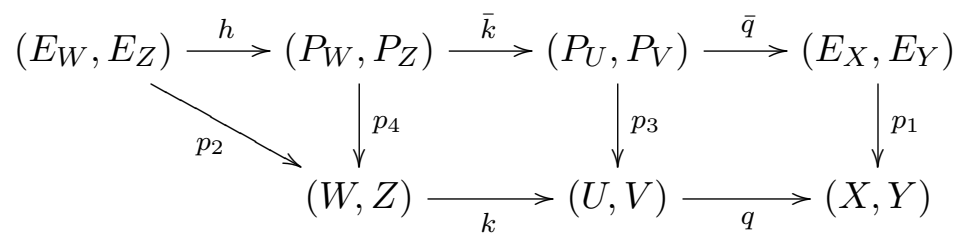

with both $p_{3}$ and $p_{4}$ also perfect fibrations of pairs, while $\bar{k}, \bar{q}$ and consequently the induced map $h$ are fiberwise homotopy equivalences.

First, we consider the case where $p_{2}$ is the pullback of $p_{1}$ and $g$ is a closed cofibration. Then the pullback construction will yield a cubical diagram of Waldhausen categories and exact functors. Two faces of this cube will commute on the nose as above, two more will commute on the nose if we choose models for pushouts (but otherwise only up to natural isomorphism compatible with restriction to subspaces), and the remaining two commute up to natural isomorphism (still compatible with restriction to subspaces) using 2.1. As a consequence, the induced square of spectra, after applying the K-theory functor to the cube and hocofib along the relevant edges, will commute up to homotopy.

Now, consider the case where $p_{2}$ is the pullback of $p_{1}$, and $g$ is a homotopy equivalence. Recall $f$ is a fiberwise homotopy equivalence so both $1 \rightarrow g^{*} g_{*}$ and $f_{*} f^{*} \rightarrow 1$ are natural homotopy equivalences on the full categories of retractive spaces. As a consequence we have

$$
f_{*} p_{2}^{*} \rightarrow f_{*} p_{2}^{*} g^{*} g_{*}=f_{*} f^{*} p_{1}^{*} g_{*} \rightarrow p_{1}^{*} g_{*}
$$

a natural homotopy equivalence between the relevant exact functors which is also compatible with restriction to subspaces. Hence, as above, the induced diagram on relative A-theory commutes up to homotopy.

Finally, we consider the case where $g$ is the identity. In this case, there is a triangular prizm of Waldhausen categories and exact functors which leads to the triangular diagram of spectra

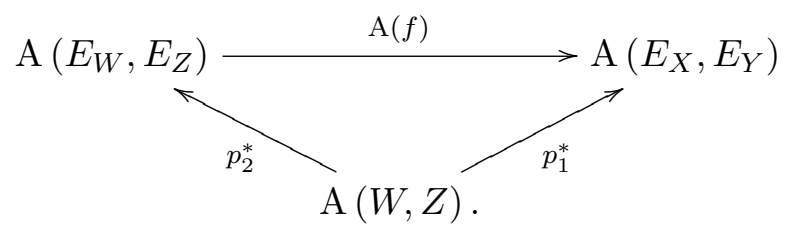


Since $f$ is a fiberwise homotopy equivalence, it again follows that $f_{*} f^{*} \rightarrow 1$ is a natural homotopy equivalence as functors on the full categories of retractive spaces. As $p_{1} \circ f=p_{2}$, this leads to a natural homotopy equivalence $f_{*} \circ p_{2}^{*}=$ $f_{*} f^{*} p_{1}^{*} \rightarrow p_{1}^{*}$, also compatible with restriction to the subspace $Z$ as desired. Once again, this leads to homotopy commutativity of the triangle of spectra, and combined with the previous cases completes the proof.

2.5. Weak Natural Transformations. We will be considering various constructions commonly viewed as equivalent in the homotopy category of spectra, also called the stable homotopy category, but we must do so with great care in order to allow us to be precise about induced maps on homotopy cofibers. As a consequence, we cannot simply work in the homotopy category of spectra, where many of these constructions are usually defined. We would prefer to use a symmetric monoidal model for the stable homotopy category, such as the S-modules of Elmendorf et al. [18] or the Symmetric Spectra of Hovey et al. [20], but will generally not need to be involved with any particular choice.

Following common usage, we have several definitions.

Definition 2.4. $\quad$ a) Given two spectra (or spaces) $Y$ and $X$, a weak map $Y \rightarrow X$ will consist of a string of morphisms of spectra (or spaces)

$$
Y=X_{0} \stackrel{f_{1}}{\longrightarrow} X_{1} \stackrel{f_{2}}{\longleftarrow} X_{2} \stackrel{f_{3}}{\longrightarrow} \ldots X_{n}=X,
$$

where each ("wrong way") map $f_{2 k}$ is a weak equivalence.

b) The weak composition of two weak maps will be defined by concatenation (possibly with an identity map added to the second string to maintain our convention that even maps are weak equivalences), even though weak maps do not form a category due to cardinality issues. Note that weak maps will determine morphisms in the homotopy category of spectra, and their weak compositions will descend to composition in the homotopy category.

c) Two weak maps will be called weakly equivalent precisely when they induce the same morphism in the homotopy category of spectra.

d) Given two spectrum-valued functors $F$ and $G$, a weak natural transformation $F \rightarrow G$ will similarly indicate a string of natural transformations

$$
F=G_{0} \stackrel{\eta_{1}}{\longrightarrow} G_{1} \stackrel{\eta_{2}}{\longleftarrow} G_{2} \stackrel{\eta_{3}}{\longrightarrow} G_{3} \ldots G_{n}=G,
$$


where each $\eta_{2 k}$ is a natural weak equivalence.

e) As above, one defines the weak composition of two weak natural transformations by concatenation, which descends to composition of natural transformations into the homotopy category of spectra.

f) Once again, two weak natural transformations will be called weakly equivalent precisely when they induce the same morphism in the homotopy category of spectra.

Remark 2.5. It follows from the definition that verifying two weak natural transformations $\eta_{n}, \ldots, \eta_{1}$ and $\nu_{n}, \ldots, \nu_{1}$ satisfy the condition that each $\eta_{i}$ is naturally (weakly) homotopy equivalent to $\nu_{i}$ will suffice to prove the weak natural transformations are weakly equivalent.

When we speak of a relative weak natural transformation below for functors from CW pairs to spectra, the naturality will be with respect to morphisms of CW pairs, which will be defined as commutative squares in topological spaces

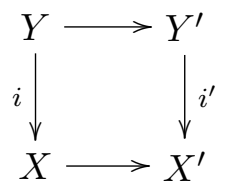

with both $i$ and $i^{\prime} \mathrm{CW}$ inclusions.

Lemma 2.6. Given a weak natural transformation of (space or) spectrum valued functors $\eta: F \rightarrow G$, there is an induced relative weak natural transformation of their extensions to $C W$ pairs, $\Psi(\eta): F \rightarrow G$.

Proof. Given a string of natural transformations

$$
F=G_{0} \stackrel{\eta_{1}}{\longrightarrow} G_{1} \prec \stackrel{\eta_{2}}{\leftarrow} G_{2} \stackrel{\eta_{3}}{\longrightarrow} \ldots G_{n}=G,
$$


we must produce a similar string for the extensions to $\mathrm{CW}$ pairs. Consider the diagram

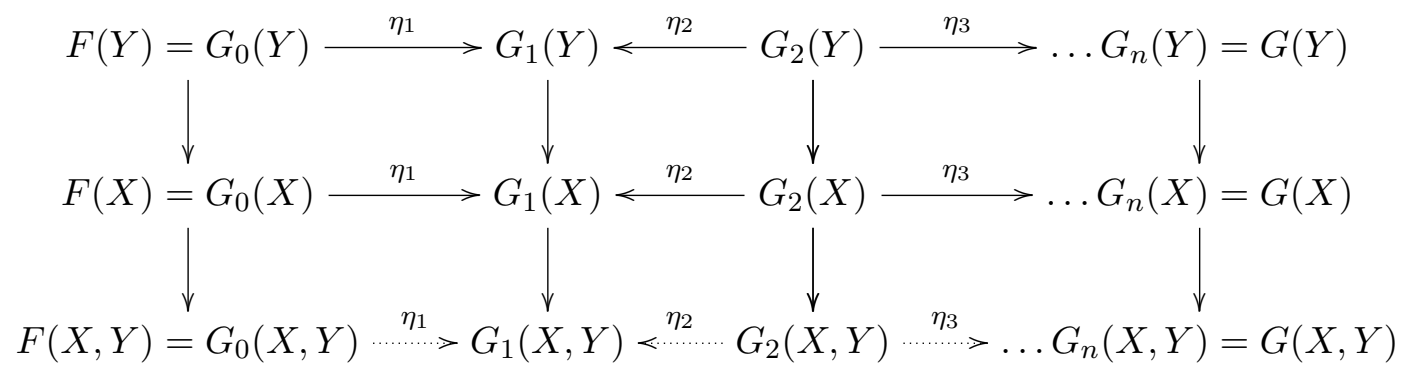

so each column is a homotopy cofiber sequence. Since the upper squares each commute by the naturality assumption on each $\eta_{i}$, the dotted arrows exist by the functoriality of this model for the homotopy cofiber. Furthermore, since $\eta_{2 k}$ is assumed to be a natural weak equivalence, we see the same remains true on the bottom row as well, which completes the definition of $\Psi(\eta)$.

Remark 2.7. The key in the previous lemma is that weak natural transformations consist of pieces which are natural on the nose, rather than up to homotopy. Thus, when forming each homotopy cofiber vertically, there is no dependence on a choice of homotopy when forming the induced map. As a consequence, there is an induced relative weak map which also does not depend on any choice of homotopy.

2.6. Constructing the (Relative) Assembly Map. Our first example of a weak natural transformation of spectrum valued functors of spaces will be the assembly map $\Sigma^{\infty}\left(X_{+}\right) \rightarrow \mathrm{A}(X)$. Here we consider the composite

$$
\Sigma^{\infty}\left(X_{+}\right) \stackrel{\simeq}{\longrightarrow} X_{+} \wedge S^{0} \stackrel{1 \wedge \mu}{\longrightarrow} X_{+} \wedge \mathrm{A}(*) \stackrel{\simeq}{\longleftarrow} \mathrm{A}^{\%}(X) \stackrel{\alpha}{\longrightarrow} \mathrm{A}(X)
$$

where $\mathrm{A}^{\%}(X)$ is defined as a certain homotopy colimit, following Weiss and Williams [35], and $\alpha$ represents their assembly map, while $\mu: S^{0} \rightarrow \mathrm{A}(*)$ is the unit map of this ring spectrum. The naturality claim follows from the fact that for a $\mathrm{CW}$ inclusion $i: Y \rightarrow X$, each square in the diagram

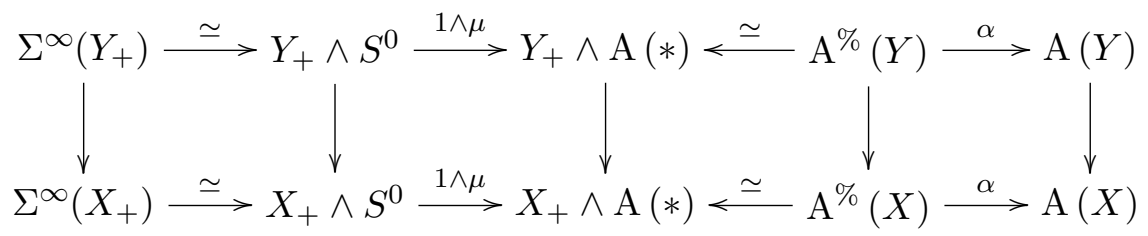


commutes.

This allows us to introduce a relative assembly map.

Corollary 2.8. There is a relative weak natural transformation of spectrum valued functors of $C W$ pairs

$$
\Psi(\alpha): \Sigma^{\infty}(X, Y) \rightarrow \mathrm{A}(X, Y)
$$

induced by the assembly map described above, via Lemma 2.6. (See Remark 2.7.)

2.7. FSP Version of A-theory. In constructing our trace map, we will use another model for the A-theory of a (connected) space based on FSPs (functors with smash products, see [7] or [8]), which comes from Waldhausen's "ring up to homotopy" approach. Recall that Kan originally constructed [22, §9], a functor from pointed connnected simplicial sets to (free) simplicial groups, the loop group, which comes equipped with a universal bundle. We will use Waldhausen's modification of the construction [34] which, among other things, makes clear that it preserves products. Hence, applying geometric realization to (the underlying simplicial set of) the loop group of the singular set of a pointed connected space $X$ defines a functor $\mathrm{G}(X)$ to topological groups (equipped with a universal $\mathrm{G}(X)$ bundle). Thus, for a connected space $X$, one chooses a basepoint and then forms the K-theory of the FSP associated to this topological monoid, $L_{\mathrm{G}(X)}(Z)=\mathrm{G}(X)_{+} \wedge Z$. For us, K-theory of an FSP is defined as

$$
\mathrm{K}(F)=\mathbb{Z} \times\left(\underset{k}{\operatorname{hocolim} \mathrm{B}}\left(\widehat{G L}_{k}(F)\right)\right)^{+} .
$$

Notice this choice of basepoint is not relevant in determining the homotopy type of the resulting K-theory space, since the loop groups for different choices of basepoint will remain isomorphic (see [34]), although the specific isomorphism depends on a choice of path between the two choices of basepoint. Thus, we will generally only have to be careful to make compatible choices of basepoints when working with maps. Of course, we are primarily interested in naturality in spaces to apply Lemma 2.6, so only for the inclusion of a $\mathrm{CW}$ pair, where basepoints can be chosen within the subspace, so this will cause no difficulty.

Notice that if $X=\coprod_{\alpha} X_{\alpha}$ is a decomposition into components, then the retractive spaces model has an isomorphism $\mathrm{A}(X) \approx \prod_{\alpha} \mathrm{A}\left(X_{\alpha}\right)$ (see e.g. [33, proof of 2.1.7]). This suggests that one extend the FSP model to arbitrary spaces by simply forming the product of the values on the components indexed on the 
set of components, which will then remain equivalent to the retractive spaces model even if $X$ is not connected.

The extended FSP model (for not necessarily connected spaces) will also be natural (with appropriate choice of a basepoint for each component), since one can extend the functoriality of the FSP model over this product construction. In particular, for $Y \rightarrow X$ a CW inclusion, even if $Y$ has more components that $X$, one has a specific map $\mathrm{A}(Y) \rightarrow \mathrm{A}(X)$ which is compatible (up to homotopy) with the comparable map on the retractive spaces model.

In what follows, we will require some notation. Given an FSP $F$, recall the matrix $\mathrm{M}_{k}(F)$ FSP defined by

$$
\mathrm{M}_{k}(F)(Z)=\operatorname{map}_{*}([k],[k] \wedge F(Z))
$$

and let $\mathcal{H}_{k}^{n}(\mathrm{G}(X))$ denote the simplicial monoid of pointed $\mathrm{G}(X)$-equivariant self homotopy equivalences of $[k] \wedge S^{n} \wedge \mathrm{G}(X)_{+}$.

Proposition 2.9. There is a weak natural transformation of spectra, where each component is a weak equivalence, $\mathrm{A}(X) \rightarrow \mathrm{K}\left(L_{\mathrm{G}(X)}\right)$.

Proof. There is a natural weak equivalence [33, Prop 2.1.4] A $(X) \rightarrow \Omega\left|h R_{h f}(*, \mathrm{G}(X))\right|$ and a natural chain of homotopy equivalences [33, Prop. 2.2 .1 and below] between $\Omega\left|h R_{h f}(*, \mathrm{G}(X))\right|$ and $\mathbb{Z} \times \lim _{n, k} \mathrm{~B}\left(\mathcal{H}_{k}^{n}(\mathrm{G}(X))\right)^{+}$.

Following Dundas, Goodwillie, and McCarthy [16, §2.3.4], we first construct a natural weak equivalence $\left(\widehat{G L}_{k}\left(L_{\mathrm{G}(X)}\right)\right)^{q} \stackrel{\sim}{\rightarrow} \operatorname{hocolim}_{x \in I}\left(\mathcal{H}_{k}^{x}(\mathrm{G}(X))\right)^{q}$ which induces an entrywise weak equivalence of simplicial spaces, and thereby a weak equivalence on realizations $\mathrm{B}\left(\widehat{G L}_{k}\left(L_{\mathrm{G}(X)}\right)\right) \stackrel{\sim}{\rightarrow} \operatorname{hocolim}_{x \in I} \mathrm{~B}\left(\mathcal{H}_{k}^{n}(\mathrm{G}(X))\right)$. The map is constructed by first commuting (unpointed) hocolim ${ }_{I}$ with the product

$$
\begin{gathered}
\left(\operatorname{hocolim}_{x \in I} \Omega^{x} \mathrm{M}_{k}\left(L_{\mathrm{G}(X)}\right)\left(S^{x}\right)\right)^{q} \\
\downarrow \\
\operatorname{hocolim}_{\left(x_{1}, \ldots, x_{q}\right) \in I^{q}}\left(\Omega^{x_{1}} \mathrm{M}_{k}\left(L_{\mathrm{G}(X)}\right)\left(S^{x_{1}}\right) \times \cdots \times \Omega^{x_{q}} \mathrm{M}_{k}\left(L_{\mathrm{G}(X)}\right)\left(S^{x_{q}}\right)\right)
\end{gathered}
$$

then including each factor in a copy of the larger stable matrix value via appropriate suspensions

$$
i_{j}: \Omega^{x_{j}} \mathrm{M}_{k}\left(L_{\mathrm{G}(X)}\right)\left(S^{x_{j}}\right) \rightarrow \Omega^{\left(x_{1}, \ldots, x_{q}\right)} \mathrm{M}_{k}\left(L_{\mathrm{G}(X)}\right)\left(S^{\left(x_{1}, \ldots, x_{q}\right.}\right)
$$


followed by the map

$$
\underset{I^{q}}{\operatorname{hocolim}} \Omega^{\left(x_{1}, \ldots, x_{q}\right)} \mathrm{M}_{k}\left(L_{\mathrm{G}(X)}\right)\left(S^{\left(x_{1}, \ldots, x_{q}\right.}\right) \rightarrow \underset{I}{\operatorname{hocolim}} \Omega^{x} \mathrm{M}_{k}\left(L_{\mathrm{G}(X)}\right)\left(S^{x}\right)
$$

induced by the natural map $I^{q} \rightarrow I$. Restricting to units up to homotopy then induces the requisite natural weak equivalence. Now we apply $\mathbb{Z} \times\left(\operatorname{hocolim}_{k} \text { ? }\right)^{+}$to get a map $\mathrm{K}\left(L_{\mathrm{G}(X)}\right) \rightarrow \mathbb{Z} \times\left(\text { hocolim }_{k} \text { hocolim }_{x \in I} \mathrm{~B}\left(\mathcal{H}_{k}^{x}(\mathrm{G}(X))\right)\right)^{+}$which we extend to $Z \times \lim _{n, k} \mathrm{~B}\left(\mathcal{H}_{k}^{n}(\mathrm{G}(X))\right)^{+}$via a natural weak map where each component is a weak equivalence, $\operatorname{hocolim}_{k} \operatorname{hocolim}_{x \in I} \mathrm{~B}\left(\mathcal{H}_{k}^{x}(\mathrm{G}(X))\right) \rightarrow \operatorname{colim}_{k, n} \mathrm{~B}\left(\mathcal{H}_{k}^{n}(\mathrm{G}(X))\right)$

2.8. Constructing the (Relative) Trace Map. The motivation for our trace map comes from the Dennis trace map in the Algebraic K-theory of rings. This is induced by a composite natural transformation

$$
\mathrm{B}\left(G L_{k}(R)\right) \stackrel{i}{\longrightarrow}\left|\mathrm{N}^{c y}\left(G L_{k}(R)\right)\right| \stackrel{m}{\longrightarrow} \mathrm{HH}\left(M_{n}(R)\right) \stackrel{\mathrm{HH}(t r)}{\longrightarrow} \mathrm{HH}(R) .
$$

Here $i$ is a canonical section $i: \mathrm{B}\left(G L_{k}(R)\right) \rightarrow\left|\mathrm{N}^{c y}\left(G L_{k}(R)\right)\right|$ of the natural projection (forgetting the zero simplex) $\pi:\left|\mathrm{N}^{c y}\left(G L_{k}(R)\right)\right| \rightarrow \mathrm{B}\left(G L_{k}(R)\right)$, with $i$ given on simplices by $\left(h_{0}, \ldots, h_{k}\right) \mapsto\left(\left(h_{0} \ldots h_{k}\right)^{-1}, h_{0}, \ldots, h_{k}\right)$, while $m$ effectively just rewrites tuples as strings of tensor products, and $\operatorname{tr}: M_{n}(R) \rightarrow R$ is the usual trace of a matrix. This yields a map $\coprod_{k \geq 0} \mathrm{~B}\left(G L_{k}(R)\right) \rightarrow \mathrm{HH}(R)$, which (after adjusting $\pi_{0}$ ), extends over the group completion to yield the Dennis trace map $\mathrm{K}(R) \rightarrow \mathrm{HH}(R)$. Unfortunately, the analog of $i$ is complicated in the case of rings up to homotopy by the fact that one must be careful when trying to invert elements which are only invertible up to homotopy.

The Bökstedt trace map [8], also published in Bökstedt, Hsiang, and Madsen [7], defines a weak natural transformation of spectra (for connected spaces) from $\mathrm{K}\left(L_{\mathrm{G}(X)}\right)$ to $t \mathrm{THH}\left(L_{\mathrm{G}(X)}\right)$, which is weakly equivalent to the stable free loop space $\Sigma^{\infty}\left(\Lambda X_{+}\right)$, and we will describe it in a moment. However, choosing the basepoint $1 \in S^{1}$ (modeled by the units of $\mathbb{C}$ ) gives a natural map, evaluation at 1, eval $1: \Sigma^{\infty}\left(\Lambda X_{+}\right) \rightarrow \Sigma^{\infty}\left(X_{+}\right)$and the (weak) composite, referred to here as the evaluation of the Bökstedt trace, is another weak natural transformation $\mathrm{K}\left(L_{\mathrm{G}(X)}\right) \rightarrow \Sigma^{\infty}\left(X_{+}\right)$(for connected spaces). For non-connected spaces, we will proceed with a product construction as discussed in subsection 2.7, keeping in mind that the natural map from a finite coproduct to the product of the same spectra is a stable homotopy equivalence. See also Madsen's discussion [27]. 
For the sake of brevity, we will follow [7] and focus on the zero spaces in constructing the Bökstedt trace map, citing compatibility with the relevant $\Gamma$-space structure to promote to the level of spectra. Recall that the space $\operatorname{THH}(F)=$ $\left|\mathrm{THH}_{\bullet}(F)\right|$, where the space of $p$-simplices of this simplicial space is

$$
\underset{I^{p+1}}{\operatorname{hocolim}} \operatorname{map}_{*}\left(S^{i_{0}} \wedge \ldots \wedge S^{i_{p}}, F\left(S^{i_{0}}\right) \wedge \ldots \wedge F\left(S^{i_{p}}\right)\right)
$$

with face maps associated to concatenation $I^{p+1} \rightarrow I^{p}$ (preceded by cyclic permuation in the last case).

Recall that for an FSP $L$, its $k$ th general linear monoid, $\widehat{G L}_{k}(L)$, is defined as the group-like topological monoid

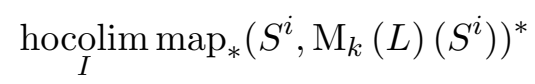

where the superscript $*$ indicates taking the subspace of homotopy units.

As usual, for a topological monoid $H, \mathrm{~B}(H)$ will refer to the realization of the simplicial space $\mathrm{N}(H)$ whose space of $p$-simplices is given by $p$-fold product of copies of $H$, with face maps given by multiplication of neighboring terms (or omission for the extremes). One defines $\mathrm{N}^{c y}(H)$ as the simplicial space whose space of $p$-simplices is given by $(p+1)$-fold product of copies of $H$, with the face map defined similarly, although the last face map is given by first cyclic permuation and then multiplying the first two terms. Notice there is a simplicial map $\pi: \mathrm{N}^{c y}(H) \rightarrow \mathrm{N}(H)$ given on simplices by projection away from the first term, which induces a composite

$$
S^{1} \times\left|\mathrm{N}^{c y}(H)\right| \rightarrow\left|\mathrm{N}^{c y}(H)\right| \rightarrow \mathrm{B}(H),
$$

where the first map is given by the $S^{1}$-action (see [7, page 472]). The adjoint map $\left|\mathrm{N}^{c y}(H)\right| \rightarrow \Lambda \mathrm{B}(H)$ is then a homotopy equivalence when $H$ is group-like.

See Goodwillie [19, I.1.8] (or Bousfield and Friedlander [9, page 311]) for the following. Let $F$ represent the associated free monoid functor, given by realizing the simplicial resolution associated to the adjunction between topological monoids and spaces, and let $H$ represent the associated topological group functor. 
Lemma 2.10. For an FSP L, there is a weak natural transformation I

$$
\begin{gathered}
\mathrm{B}\left(\widehat{G L}_{k}(L)\right) \longleftarrow \simeq \mathrm{B}\left(F \widehat{G L}_{k}(L)\right) \stackrel{\simeq}{\longrightarrow} \mathrm{B}\left(H \widehat{G L}_{k}(L)\right) \\
\left|\mathrm{N}^{c y}\left(\widehat{G L}_{k}(L)\right)\right| \stackrel{\downarrow^{i}}{\simeq}\left|\mathrm{N}^{c y}\left(F \widehat{G L}_{k}(L)\right)\right| \stackrel{\simeq}{\longrightarrow}\left|\mathrm{N}^{c y}\left(H \widehat{G L}_{k}(L)\right)\right|
\end{gathered}
$$

which acts as a section, in the homotopy category of spaces, of the natural projection $\pi:\left|\mathrm{N}^{c y}\left(\widehat{G L}_{k}(L)\right)\right| \rightarrow \mathrm{B}\left(\widehat{G L}_{k}(L)\right)$.

Moving on toward defining the trace, there is a straightforward map

$$
M:\left|\mathrm{N}^{c y}\left(\widehat{G L}_{k}(L)\right)\right| \rightarrow \operatorname{THH}\left(\mathrm{M}_{k}(L)\right)
$$

induced by (including and) multiplying at the level of simplices. Next we have the Morita equivalence map following [7, (3.9.3) on page 480] as always with $r=1$ since we are not after $C_{r}$-equivariant results. There is also a nice description of this map due to Schlichtkrull [28], using a slightly different definition of THH.

Lemma 2.11. There is a weak natural transformation of spaces for any FSP L,

$$
\operatorname{THH}\left(\mathrm{M}_{k}(L)\right) \longleftarrow\left\|X_{\bullet, \bullet}(1)\right\| \longrightarrow \operatorname{THH}(L),
$$

where both natural transformations are natural weak equivalences.

Hence, the weak composite

$$
\mathrm{B}\left(\widehat{G L}_{k}(L)\right) \rightarrow\left|\mathrm{N}^{c y}\left(\widehat{G L}_{k}(L)\right)\right| \rightarrow \mathrm{THH}\left(\mathrm{M}_{k}(L)\right) \rightarrow \mathrm{THH}(L)
$$

gives a weak natural transformation, as space valued functors from FSPs. Of course, we then promote to a weak natural transformation of spectra by noting all of the above is compatible with $\Gamma$-space structures, as described in [7, §4]. Choosing the FSP $L_{\mathrm{G}(X)}$ to be multiplication by the (pointed version of the) topological group $\mathrm{G}(X)$ (coming from the Kan loop group functor, see subsection 2.7 ), we then have a composite weak natural transformation as functors from spaces (after careful choices of a basepoint for every component) to spectra

$$
\mathrm{K}\left(L_{\mathrm{G}(X)}\right) \rightarrow t \mathrm{THH}\left(\mathrm{M}_{k}\left(L_{\mathrm{G}(X)}\right)\right) \rightarrow t \operatorname{THH}\left(L_{\mathrm{G}(X)}\right) .
$$

As described above, by $\mathrm{G}(X)$ a group-like topological group and a loop group for $X$, there is a natural weak equivalence

$$
\left|\mathrm{N}^{c y}(\mathrm{G}(X))\right| \rightarrow \Lambda \mathrm{B}(\mathrm{G}(X)) \rightarrow \Lambda X .
$$


See [7, Prop. 3.7] (with $r=1$ ) for details, keeping in mind that we again promote from spaces to spectra by compatibility with the $\Gamma$-space structures.

Lemma 2.12. There is a weak natural transformation of spectra

$$
\operatorname{THH}\left(L_{\mathrm{G}(X)}\right) \stackrel{\sim}{\rightarrow} \Sigma^{\infty}\left(\Lambda \mathrm{B}(\mathrm{G}(X))_{+}\right) \stackrel{\sim}{\rightarrow} \Sigma^{\infty}\left(\Lambda X_{+}\right)
$$

where each component map is a natural weak equivalence.

Combining the results of this subsection and the last then yields the following:

Proposition 2.13. There is a weak natural transformation of functors from spaces (after appropriate choices of basepoints for components) to spectra

$$
\begin{aligned}
& \mathrm{A}(X) \longrightarrow \mathrm{K}\left(L_{\mathrm{G}(X)}\right) \longrightarrow t \operatorname{THH}\left(\mathrm{M}_{k}\left(L_{\mathrm{G}(X)}\right)\right) \longrightarrow t \mathrm{THH}\left(L_{\mathrm{G}(X)}\right) \\
& \Sigma^{\infty}\left(X_{+}\right) \longleftarrow{ }_{e v a l_{1}} \Sigma^{\infty}\left(\Lambda X_{+}\right),
\end{aligned}
$$

which we refer to as the evaluation of the (extended) Bökstedt trace and denote tr.

Now we can define the relative trace map as the relative weak natural transformation associated to tr, using Lemma 2.6. (See Remark 2.7.)

Corollary 2.14. There is a relative weak natural transformation, called the relative trace map, $\Psi(\operatorname{tr}): \mathrm{A}(X, Y) \rightarrow \Sigma^{\infty}(X, Y)$.

Once again, this will be natural only with respect to maps of CW pairs.

2.9. The Candidate for the Transfer. Since the axioms of Becker and Schultz work with a transfer of pairs, we have introduced relative versions of the assembly, A-theory transfer, and the evaluation of the Bökstedt trace map. We now have three weak natural transformations given a perfect fibration of pairs $\left(p_{X}, p_{Y}\right)$ : $\left(E_{X}, E_{Y}\right) \rightarrow(X, Y)$ and we take their (weak) composite to form our expected weak map $\mathbb{T}\left(p, E_{X}, E_{Y}\right)$ :

$$
\Sigma^{\infty}(X, Y) \stackrel{\Psi(\alpha)}{\longrightarrow} \mathrm{A}(X, Y) \stackrel{\tau_{\mathrm{A}}\left(p_{X}, p_{Y}\right)}{\longrightarrow} \mathrm{A}\left(E_{X}, E_{Y}\right) \stackrel{\Psi(\operatorname{tr})}{\longrightarrow} \Sigma^{\infty}\left(E_{X}, E_{Y}\right)
$$

which we abbreviate as $\mathbb{T}$. 
For the convenience of the reader, we will next state the Becker-Schultz axioms and their main theorem in the current language. The statement of the additivity axiom requires reference to sums of maps, which we will always perform using the $H$-space operation from the last loop space operation being performed in the usual colim $\Omega^{n} \Sigma^{n} E$. Our difference operation will then come from reversing the direction of the loop in the second coordinate. These choices become irrelevant in the homotopy category, hence are only required to make our statements welldefined with respect to weak maps.

Definition 2.15. A transfer is a function which assigns to each perfect fibration of pairs $p:\left(E_{X}, E_{Y}\right) \rightarrow(X, Y)$ a weak map

$$
\mathbb{T}\left(p, E_{X}, E_{Y}\right): \Sigma^{\infty}(X, Y) \rightarrow \Sigma^{\infty}\left(E_{X}, E_{Y}\right)
$$

satisfying:

- Naturality: If $f$ is a fiberwise homotopy equivalence covering the map of CW pairs $g:(W, Z) \rightarrow(X, Y)$, then $\mathbb{T}\left(p_{1}, E_{X}, E_{Y}\right) \circ \Sigma^{\infty}(g)$ and $\Sigma^{\infty}(f) \circ$ $\mathbb{T}\left(p_{2}, E_{W}, E_{Z}\right)$ are weakly equivalent (as weak maps, see $\left.2.4(\mathrm{c})\right)$.

- Normalization: $\mathbb{T}(1, X, Y)$ is weakly equivalent to the identity (as a weak map).

- Multiplicativity: Given two perfect fibrations of pairs $p_{1}:\left(E_{X}, E_{Y}\right) \rightarrow$ $(X, Y)$ and $p_{2}:\left(E_{W}, E_{Z}\right) \rightarrow(W, Z)$, the diagram

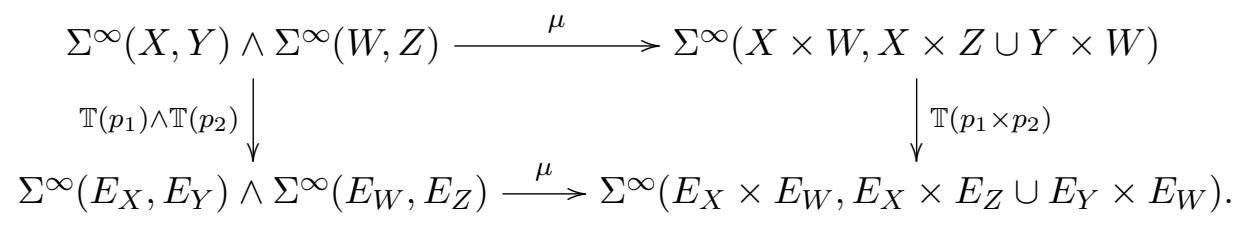

commutes up to a natural homotopy in the category of spectra.

- Strong Additivity: Assume

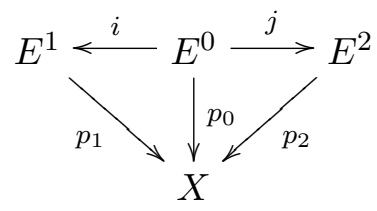

is a diagram of perfect fibrations, with $p: E_{X} \rightarrow X$ the pushout (perfect) fibration and $i$ a cofibration, while $(X, Y)$ is a CW-pair. For $n=$ $0,1,2$, let $k^{n}:\left(E_{X}^{n}, E_{Y}^{n}\right) \rightarrow\left(E_{X}, E_{Y}\right)$ denote the relevant inclusion and $p_{n}:\left(E_{X}^{n}, E_{Y}^{n}\right) \rightarrow(X, Y)$ the perfect fibration of pairs. Then $\mathbb{T}(p)$ and 
$\Sigma^{\infty}\left(k^{1}\right) \mathbb{T}\left(p_{1}\right)+\Sigma^{\infty}\left(k^{2}\right) \mathbb{T}\left(p_{2}\right)-\Sigma^{\infty}\left(k^{0}\right) \mathbb{T}\left(p_{0}\right)$ are weakly equivalent (as weak maps).

Theorem 2.16. Any transfer (in the sense of Def. 2.15) is weakly equivalent (as a weak map, see 2.4(c)) to the Becker-Gottlieb transfer when evaluated at any compact ANR fibration over a finite base.

Proof. This is merely a restatement of the Main Theorem of [6] into the language of weak maps. Since a transfer as in 2.15 induces a bundle transfer in the notation of [6] satisfying axioms I-III and $\mathrm{IV}^{+}$, their result implies the induced map in the homotopy category is the Becker-Gottlieb transfer. As the construction of the Becker-Gottlieb transfer in either [4] or [5] does produce a weak map, it then suffices to recall our convention that weak maps be called weakly equivalent when they induce the same map in the homotopy category.

We can now state our main result:

Theorem 2.17. Suppose $X$ is a finite $C W$ complex and $p: E \rightarrow X$ is a compact ANR fibration. Then the evaluation of the Bökstedt trace of the algebraic K-theory transfer of $p$ is weakly equivalent (as a weak map, see 2.4(c)) to the BeckerGottlieb transfer of $p$, that is $\operatorname{tr} \circ \tau_{\mathrm{K}}(p)=\tau_{B G}(p)$ in the homotopy category of spectra.

Proof. As indicated above, our method of proof is to verify the axioms of Becker and Schultz for $\mathbb{T}(p)$ (assuming only that $p$ is a perfect fibration with finite base). We verify the naturality and normalization axioms in Props 4.1 and 4.2, the multiplicativity axiom in Prop 3.8 and the strong additivity axiom in Prop 5.3 .

\section{MultiplicAtivity}

The point here is to show that all three (relative) components of the candidate transfer $\mathbb{T}$ are compatible with the external pairing on relative A-theory.

3.1. Multiplicativity of the A-theory transfer. We begin by introducing relative pairings and our desired notion of multiplicative, keeping in mind the material from subsection 2.5 on induced relative functors, denoted there with a $\Psi$. 
Definition 3.1. When each intermediate spectrum-valued functor in a weak natural transformation (in the sense of subsection 2.6) is equipped with an external pairing, such that each diagram of the form

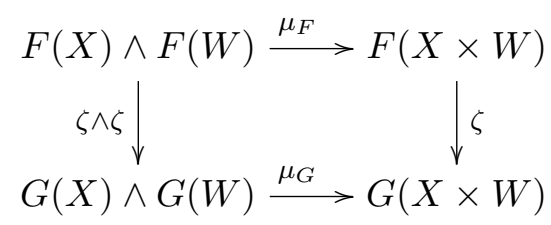

commutes up to a homotopy natural in each variable, it will be referred to as a multiplicative weak natural transformation.

Lemma 3.2. Let $F$ be a spectrum-valued functor of spaces, equipped with an external pairing that is natural up to a natural homotopy. Then the external product for the functor $F$ induces a relative external product

$$
\mu_{r e l}: F(X, Y) \wedge F(W, Z) \rightarrow F\left(X \times W, X \times Z \cup_{Y \times Z} Y \times W\right)
$$

which is also natural up to a natural homotopy.

Proof. First, notice the left hand side is naturally homotopy equivalent to the homotopy pushout of the diagram

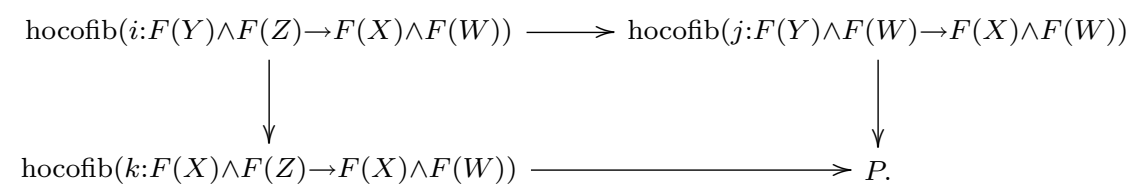

Now notice

$$
F\left(X \times W, X \times Z \cup_{Y \times Z} Y \times W\right)
$$

is also defined as a homotopy cofiber. Furthermore, from all three homotopy cofibers in the homotopy pushout diagram above, the maps from the targets of $i, j$, and $k$

$$
\mu: F(X) \wedge F(W) \rightarrow F(X \times W)
$$

are the same. Thus, it will suffice to verify that the three composite maps $\mu \circ j$, $\mu \circ i$ and $\mu \circ k$ each factor up to homotopy through the map

$$
F\left(X \times Z \cup_{Y \times Z} Y \times W\right) \rightarrow F(X \times W) .
$$

This follows from naturality of the homotopy for the external product of $F$ and the commutative pushout diagram which defines the union. 
The key application is the relative pairing in A-theory that follows.

Corollary 3.3. The external smash product of retractive spaces induces an external pairing on relative A-theory

$$
\mu: \mathrm{A}(X, Y) \wedge \mathrm{A}(W, Z) \rightarrow \mathrm{A}\left(X \times W, X \times Z \cup_{Y \times Z} Y \times W\right) .
$$

Proof. By Lemma 3.2, it will suffice to show the diagram of Waldhausen categories and (bi-)exact functors

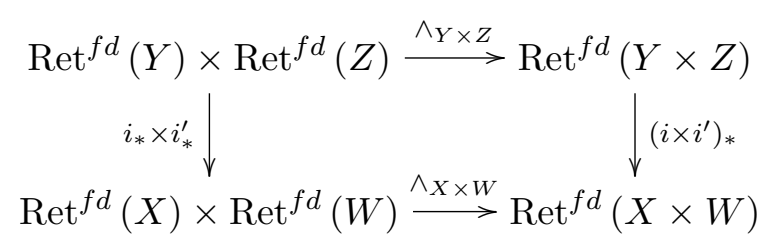

commutes up to a unique isomorphism.

Recall product with a fixed space preserves pushouts (as we work with compactly generated spaces), and the universal property of pushouts implies there are unique isomorphisms $i_{*} Y \rightarrow X, i_{*}^{\prime} Z \rightarrow W$, and $\left(i \times i^{\prime}\right)_{*}(Y \times Z) \rightarrow X \times W$. Hence, for any pair $U \in \operatorname{Ret}^{f d}(Y)$ and $V \in \operatorname{Ret}^{f d}(Z)$, there is also a unique isomorphism

$$
i_{*} U_{X} \vee_{W} i_{*}^{\prime} V \rightarrow\left(i \times i^{\prime}\right)_{*}\left(U_{Y} \vee_{Z} V\right)
$$

by inspection of the defining pushouts (see subsection 2.2). Now by inspection of the defining pushout diagrams, one has unique isomorphisms in all three input slots, hence the required unique isomorphism

$$
i_{*} U_{X} \wedge_{W} i_{*}^{\prime} V \rightarrow\left(i \times i^{\prime}\right)_{*}\left(U_{Y} \wedge_{Z} V\right)
$$

Lemma 3.4. Given two perfect fibrations of pairs $p_{1}:\left(E_{X}, E_{Y}\right) \rightarrow(X, Y)$ and $p_{2}:\left(E_{W}, E_{Z}\right) \rightarrow(W, Z)$, the diagram

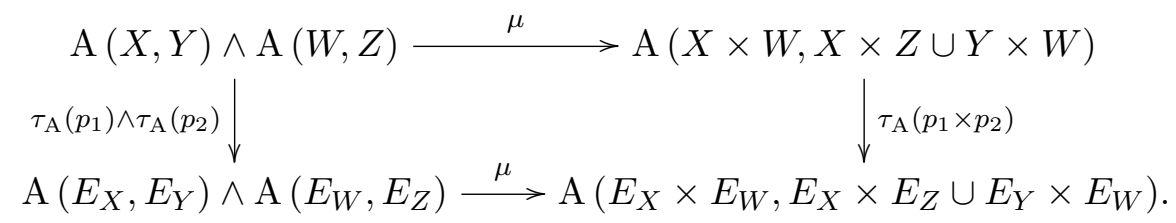

commutes up to a natural homotopy in the category of spectra. 
Proof. Since $\Omega\left|N w \delta_{\bullet}(?)\right|$ preserves products up to isomorphism, it would suffice to verify that the following diagram of (bi-)exact functors commutes up to a unique natural isomorphism (which as a consequence will be compatible with restriction to subspaces):

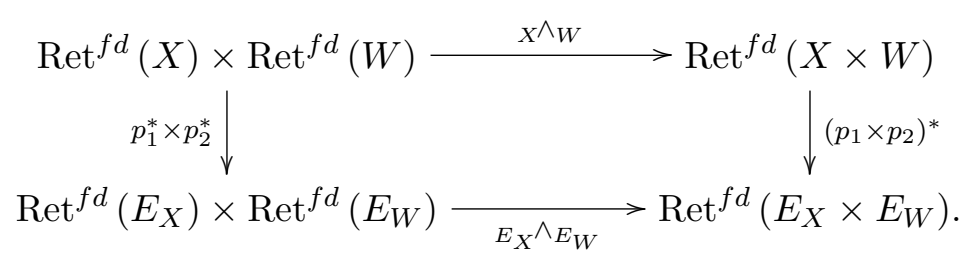

Choose $U \in \operatorname{Ret}^{f d}(X)$ and $V \in \operatorname{Ret}^{f d}(W)$. Since $X \longmapsto U$ and $W \longmapsto V$ are cofibrations, we see

$$
U \times W \cup_{X \times W} X \times V \longmapsto U \times V
$$

is also a cofibration. Hence, we again use Lemma 2.1 to see there is a unique natural isomorphism in $\operatorname{Ret}^{f d}\left(E_{X} \times E_{W}\right)$

$$
p_{1}^{*}(U)_{E_{X}} \wedge_{E_{W}} p_{2}^{*}(V) \cong\left(p_{1} \times p_{2}\right)^{*}\left(U_{X} \wedge_{W} V\right) .
$$

\subsection{Multiplicative Relative Weak Natural Transformations.}

Definition 3.5. An induced relative weak natural transformation $\Psi(\zeta)$ will be called multiplicative if for each component natural transformation $\zeta: F \rightarrow G$ the source and target are equipped with external pairings and the following diagram commutes up to a homotopy which is natural in each variable:

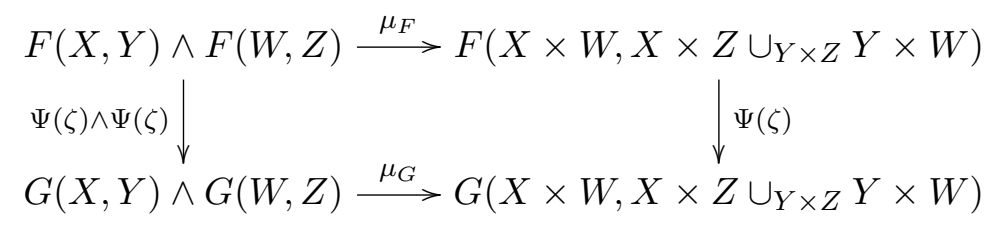

The key general result in this direction is the following:

Lemma 3.6. Suppose $\zeta: F \rightarrow G$ is a multiplicative weak natural transformation (in the sense of Def. 3.1). Then $\Psi(\zeta)$, the relative weak natural transformation it induces, is multiplicative (in the sense of Def. 3.5). 
Proof. As in the proof of Lemma 3.2, recall $F(X, Y) \wedge F(W, Z)$ is naturally homotopy equivalent to a homotopy pushout, as is $G(X, Y) \wedge G(W, Z)$. Thus, it will suffice to show the two maps

$$
\operatorname{hocofib}(F(Y) \wedge F(W) \rightarrow F(X) \wedge F(W)) \rightarrow G\left(X \times W, X \times Z \cup_{Y \times Z} Y \times W\right)
$$

are homotopic in a natural way, and similarly for $F(X) \wedge F(Z)$. Notice that each of these maps factors through $G(X \times W, Y \times W)$, so it will suffice to see they are naturally homotopic up to that point. Since naturality of this homotopy will imply compatibility with the "restriction" to

$$
\operatorname{hocofib}(F(Y) \wedge F(Z) \rightarrow F(X) \wedge F(W)),
$$

this will suffice to complete the proof.

Now recall that a map from one homotopy cofiber of this form to another is completely determined by the corresponding null-homotopy of the composite

$$
F(Y) \wedge F(W) \rightarrow G(Y \times W) \rightarrow G(X \times W) .
$$

Thus, it will suffice to see the two null-homotopies are themselves naturally homotopic. In the case of the first portion of $\mu_{G} \circ(\zeta \wedge \zeta)$, the null homotopy will arise by applying the reduced cone operator (smashing the composite map with the unit interval, using one end as basepoint) to

$$
F(Y) \wedge F(W) \rightarrow G(Y) \wedge G(W) \rightarrow G(Y \times W)
$$

and then following with the chosen null homotopy of $G(Y \times W) \rightarrow G(X \times W)$. For the first portion of $\zeta \circ \mu_{F}$, the construction is similar using the composite

$$
F(Y) \wedge F(W) \rightarrow F(Y \times W) \rightarrow G(Y \times W) .
$$

Since these two composites are assumed to be naturally homotopic by the assumption that $\zeta$ is a multiplicative weak natural transformation, we may extend this natural homotopy over the cones to show the two induced null homotopies of

$$
F(Y) \wedge F(W) \rightarrow G(Y \times W) \rightarrow G(X \times W)
$$

are naturally homotopic, which suffices to complete the proof as indicated above.

Proposition 3.7. Both the assembly map and the evaluation of the Bökstedt trace map are multiplicative weak natural transformations of spectra. 
Proof. This is a tedious business of inspecting each commutative square involved in the construction of the assembly map (see subsection 2.6) and in the construction of the transfer map (see subsection 2.8).

For the assembly map, the first three functors involved all have external pairing maps induced from natural homotopy equivalences on the variables together with a ring spectrum product, which makes the condition simple to verify. The remaining two portions come from inspecting the external pairing on $A^{\%}($ ?), which comes from the external smash product of retractive spaces over simplices. Taken together, this implies the assembly map is a multiplicative weak natural transformation.

For the trace map, we begin by verifying that the change of A-theory models from the retractive spaces model to the FSP model is multiplicative:

Recall the transition (of Waldhausen's [30]) from the retractive spaces over $X$ model to the free (pointed) $\mathrm{G}(X)$-spaces model takes a retractive space $W$ over $X$ to $p_{1}^{*}(W) / P_{X}$, where $P_{X}$ is a universal $\mathrm{G}(X)$ bundle over $X$. In the retractive spaces model the external smash product induces the external pairing, while the ordinary smash product induces the external pairing in the free $\mathrm{G}(X)$-spaces model. Since $P_{X \times Y}$ is homeomorphic to $P_{X} \times P_{Y}$, taking the external smash product of $p_{1}^{*}(W)$ and $p_{2}^{*}(V)$ and then collapsing $P_{X \times Y}$ will be homeomorphic to taking the smash product of collapsing $P_{X}$ in $p_{1}^{*}(W)$ and $P_{Y}$ in $p_{1}^{*}(V)$ separately. This will give a natural homeomorphism at the level of models, hence a natural homotopy at the level of A-theory spectra, since these transitions are all given by exact functors.

For the remaining steps in the passage from the free $\mathrm{G}(X)$-spaces model to Waldhausen's version of the FSP model, at each step the external pairing is induced by the ordinary smash product of pointed spaces. Thus, an inspection of the chain of weak homotopy equivalences in a proof of Waldhausen [30, Theorem 2.2.1, pages 386 through 388] leads to the conclusion that the transitions between various models are all compatible (up to a homotopy natural in each variable) with external pairings in the homotopy category of spectra.

For the passage from Waldhausen's version of the FSP model to $\mathrm{K}\left(L_{\mathrm{G}(X)}\right)$, consider the following. The naturality of $\mathrm{G}(X)$ yields a natural map $\mathrm{G}(X \times Y) \rightarrow$ $\mathrm{G}(X) \times \mathrm{G}(Y)$ which is an isomorphism by inspection [34] and induces the external pairings in question (see $[31,(1.3)]$ ). For FSPs, this gives a pairing (in the sense 
of [7])

$$
L_{\mathrm{G}(X)} \wedge L_{\mathrm{G}(Y)} \rightarrow L_{\mathrm{G}(X) \times \mathrm{G}(Y)}
$$

whose target is canonically identified to $L_{\mathrm{G}(X \times Y)}$ by the isomorphism of topological groups. Careful inspection of the construction at the heart of the proof of Prop 2.9 then verifies the resulting external pairing is natural on the level of spaces. In order to promote to spectra, as we will continue to do below, one then verifies all constructions are compatible with the $\Gamma$-space structures defined in $[16, \S 2.3 .4]$ (or later, in $[7, \S 4]$ ).

Next Bökstedt, Hsiang, and Madsen [7, page 503] point out that the Bökstedt trace map from the FSP model for A-theory to $t \mathrm{THH}$ is multiplicative in the homotopy category of spectra. Since their pairings are all induced by the pairing of FSPs indicated above, it is simply a tedious verification to see the relevant homotopy can be chosen to be natural in each variable.

For the passage from THH to the stable free loop space, notice the interlacing of spheres built into the external product on THH reduces this portion to verifying that the map $\left|\mathrm{N}^{c y}\left(\Gamma_{X}\right)\right| \rightarrow \Lambda\left|\mathrm{N}\left(\Gamma_{X}\right)\right|$ is multiplicative as in [7, page 472]. To see the remaining portion, notice the standard $S^{1}$ action on a product of cyclic nerves is the diagonal action, where $S^{1}$ acts separately on each factor, and this is compatible with the usual action on the cyclic nerve of a product. Thus, taking the adjoint yields a product of maps as above, compatible with the adjoint map for the product FSP. Thus, the map from THH to the stable free loop space is multiplicative up to a natural homotopy.

Finally, evaluation at the unit of $\mathbb{C}$ will be multiplicative on the level of spaces by construction, and the stabilization preserves smash products up to natural isomorphism. Thus, the long composite is, in fact, a multiplicative weak natural transformation.

Combining the last two results now gives us what we will need below.

Proposition 3.8. Given two perfect fibrations of pairs $p_{1}:\left(E_{X}, E_{Y}\right) \rightarrow(X, Y)$ and $p_{2}:\left(E_{W}, E_{Z}\right) \rightarrow(W, Z)$, the diagram

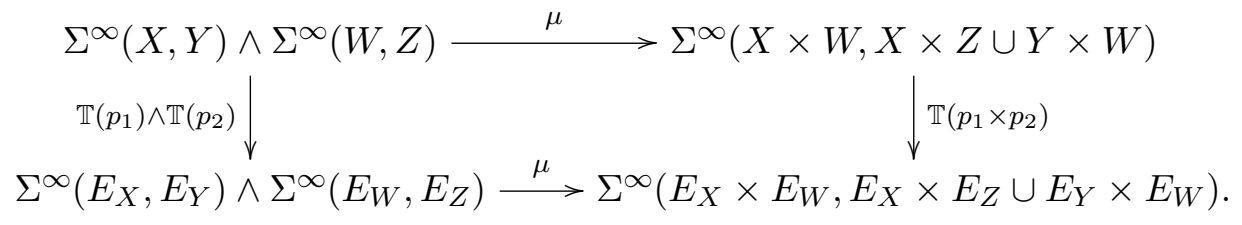


commutes up to a natural homotopy in the category of spectra.

Proof. Notice $\Psi(\alpha)$ and $\Psi(\operatorname{tr})$ are multiplicative relative weak natural transformations by Lemma 3.6 and Prop 3.7, while the multiplicativity of the relative A-theory transfer is verified in Lemma 3.4 .

\section{NATURAlity AND Normalization}

This section contains two propositions, dealing with each of the axioms listed in the section title for our candidate transfer $\mathbb{T}$. With the technical preliminaries handled in section 2, each of these is straightforward, unlike the remaining strong additivity axiom which is considered in the last section.

First, we establish the naturality property of $\mathbb{T}$.

Proposition 4.1. Suppose $f: E_{W} \rightarrow E_{X}$ is a fiberwise homotopy equivalence making the diagram

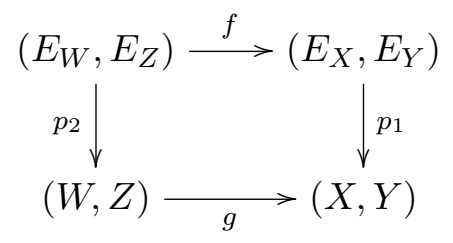

commute. Then the diagram

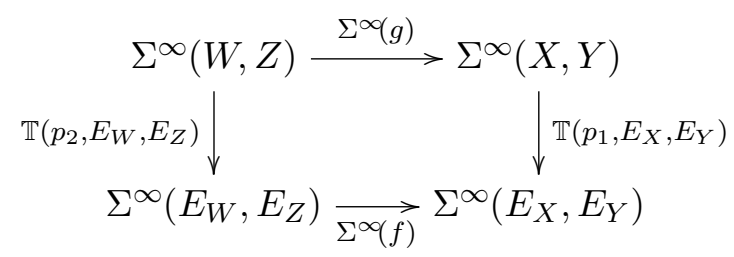

commutes in the homotopy category of spectra (or equivalently, the composites are weakly equivalent as weak maps).

Proof. This breaks up into a series of commutative diagrams in the homotopy category of spectra. Commutativity of

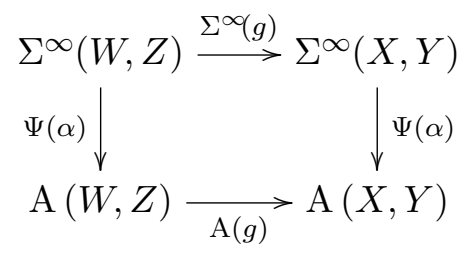


up to homotopy is the naturality of the relative assembly map from Cor 2.8 , since $g$ is a map of pairs. Then homotopy commutativity of the diagram

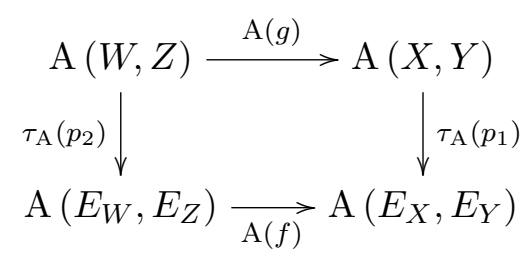

follows by Lemma 2.3. Finally, we have the homotopy commutative diagram

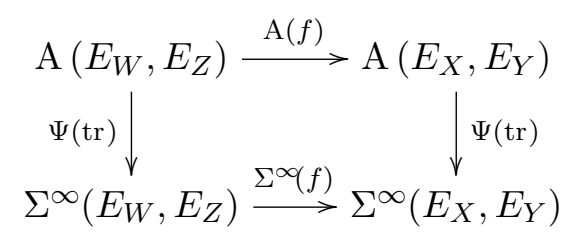

given by the naturality of the relative trace map from Cor 2.14 , since $f$ is also a map of pairs.

Now we have the normalization axiom for $\mathbb{T}$.

Proposition 4.2. For the identity map $1:(X, Y) \rightarrow(X, Y)$, the weak map

$$
\mathbb{T}(1, X, Y): \Sigma^{\infty}(X, Y) \rightarrow \Sigma^{\infty}(X, Y) .
$$

is weakly equivalent to the identity.

Proof. Since the relative A-theory transfer of the identity fibration is clearly equivalent to the identity by construction, it suffices to see that

$$
\Sigma^{\infty}(X, Y) \stackrel{\alpha}{\rightarrow} \mathrm{A}(X, Y) \stackrel{\operatorname{tr}}{\rightarrow} \Sigma^{\infty}(X, Y)
$$

is the identity up to a natural weak equivalence.

However, in the absolute case Waldhausen shows [31, Theorem 5.1]

$$
\Sigma^{\infty}\left(X_{+}\right) \stackrel{\alpha}{\rightarrow} \mathrm{A}(X) \stackrel{\operatorname{tr}}{\rightarrow} \Sigma^{\infty}\left(X_{+}\right)
$$

is naturally weakly equivalent to the identity on $\Sigma^{\infty}\left(X_{+}\right)$(as a map of spectra by his Remark 5.3), which implies the same is true with $Y$ in place of $X$ everywhere. Hence naturality of this weak equivalence implies $\Psi(\operatorname{tr} \alpha) \sim \Psi(\operatorname{tr}) \Psi(\alpha) \sim$ $\mathbb{T}(1, X, Y)$ is naturally weakly equivalent to the identity as well. 


\section{Strong AdDitivity}

We now move on to the most subtle of the axioms for our composite, the strong additivity axiom, verified in Proposition 5.3. One would like to simply appeal to the additivity of the evaluation of the Bökstedt trace and additivity of the algebraic K-theory transfer map. Unfortunately, the homotopy constructed by the first author [13] to verify the additivity of the algebraic K-theory transfer need not be natural, because of its reliance upon Waldhausen's Additivity Theorem [30, Thm 1.4.2] which makes no claim of naturality. Thus, to prove additivity for the algebraic K-theory transfer map in the relative case, we must verify the existence of compatible homotopies for the sub-fibration and the original fibration. Throughout this section we will be working with retractive spaces, with notation established in section 2 .

As referred to in subsection 2.3, Williams [36] uses the homotopy (parametrized) Euler characteristic to give lifts of transfer maps for the algebraic K-theory of spaces to his bivariant A-theory. Thus, our required homotopies may be produced by constructing paths in this bivariant A-theory space between points associated to homotopy Euler characteristics, which is the technique used by the first author in [13]. We will expand upon this technique, using a different map out of the bivariant A-theory, to produce our homotopy for the sub-fibration so that it will be compatible with that for the original fibration.

We begin by introducing some notation we will use throughout this section.

We will assume

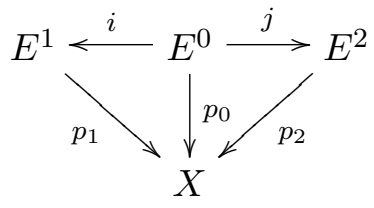

is a diagram of perfect fibrations, with $p_{X}: E_{X} \rightarrow X$ the pushout (perfect) fibration and $i$ a cofibration, while $(X, Y)$ is a CW-pair. For $n=0,1,2$, let $k^{n}$ : $\left(E_{X}^{n}, E_{Y}^{n}\right) \rightarrow\left(E_{X}, E_{Y}\right)$ denote the relevant inclusion and $p_{n}:\left(E_{X}^{n}, E_{Y}^{n}\right) \rightarrow(X, Y)$ the perfect fibration of pairs.

The heart of our additivity result is the A-theory Transfer Additivity Theorem of the first author [13], which suggests the following definition. Recall the sum 
operation used here comes from the coproduct operation

$$
(W, Z) \mapsto W \sqcup Z
$$

in the category of retractive spaces, while the difference operation is instead induced by

$$
(W, Z) \mapsto W \sqcup \Sigma Z
$$

where $\Sigma$ here indicates the fiberwise suspension in the category of retractive spaces.

Definition 5.1. Given a decomposed perfect fibration $p_{X}$ as above, we will let $p_{\operatorname{dec}(X)}^{*}: \mathrm{A}(X) \rightarrow \mathrm{A}\left(E_{X}\right)$ denote the sum (indicated above) of maps

$$
\mathrm{A}\left(k^{1}\right) p_{1}^{*}+\mathrm{A}\left(k^{2}\right) p_{2}^{*}-\mathrm{A}\left(k^{0}\right) p_{0}^{*} .
$$

Theorem 5.2 (Transfer Additivity Theorem of Dorabiała [13]). For a decomposed perfect fibration $p_{X}$ as above, there is a homotopy in the category of spectra

$$
p_{X}^{*} \simeq \mathrm{A}\left(k^{1}\right) p_{1}^{*}+\mathrm{A}\left(k^{2}\right) p_{2}^{*}-\mathrm{A}\left(k^{0}\right) p_{0}^{*}=p_{\operatorname{dec}(X)}^{*} .
$$

Given a CW-pair $(X, Y)$ as above, we would like to define the decomposed transfer of the perfect fibration of pairs. Thus, we first note that there is a natural isomorphism (at the level of retractive spaces) between the composites in the diagram

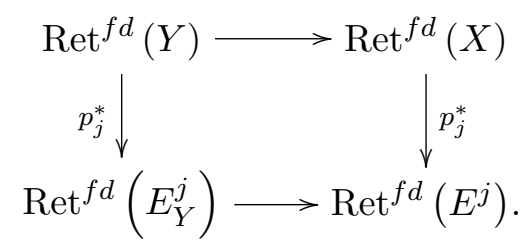

As a consequence, we may define the decomposed transfer of the perfect fibration of pairs as the homotopy class of maps induced (as in Def. 2.2) from the corresponding homotopy in the diagram of spectra below.

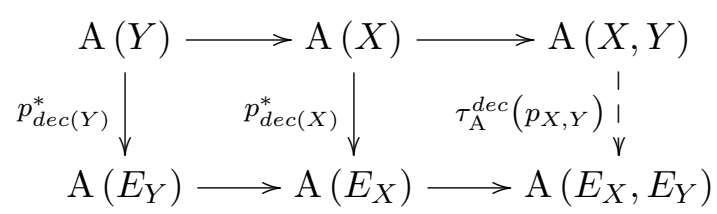

We will verify that the homotopy Euler characteristic, considered as a point in the zero space of the bivariant A-theory spectrum, is a lift of $p_{X}^{*} \alpha$ (see Lemma 
5.5) over a certain map of spectra

$$
\mathrm{A}\left(\begin{array}{l}
E_{X} \\
\downarrow \\
D_{X}
\end{array}\right) \rightarrow \operatorname{map}\left(X, \mathrm{~A}\left(E_{X}\right)\right)
$$

Similarly, one can define (see $[13$, page 260$])$ a point $\chi\left(p_{\operatorname{dec}(X)}\right)$ in $\Omega^{\infty} \mathrm{A}\left(\begin{array}{l}E_{X} \\ \downarrow \\ X\end{array} p_{X}\right)$ which is a lift of $p_{\operatorname{dec}(X)}^{*} \alpha$ over the displayed map. These are the same points in $\mathrm{A}\left(\begin{array}{c}E_{X} \\ \downarrow_{X} p_{X} \\ X\end{array}\right)$ shown to be connected by a (not neccesarily natural) path in [13], and we will label that path $\gamma_{X}$. Williams's bivariant theory then comes equipped with a restriction map Res $: \mathrm{A}\left(\begin{array}{c}E_{X} \\ \downarrow \\ p_{X} \\ X\end{array}\right) \rightarrow \mathrm{A}\left(\begin{array}{c}E_{Y} \\ \downarrow \\ \downarrow\end{array} p_{Y}\right)$ coming from the pullback diagram defining a sub-fibration. However, it is not clear that $\operatorname{Res}\left(\gamma_{X}\right)=\gamma_{Y}$, although there is a natural path $\beta_{X, Y}$ from $\operatorname{Res}\left(\chi\left(p_{X}\right)\right)$ to $\chi\left(p_{Y}\right)$ (since they correspond to retractive spaces which are naturally homeomorphic). One also has a similar path $\beta_{\operatorname{dec}(X, Y)}$ from $\operatorname{Res}\left(\chi\left(p_{\operatorname{dec}(X)}\right)\right)$ to $\chi\left(p_{\operatorname{dec}(Y)}\right)$, again corresponding to a natural homeomorphism of retractive spaces. Thus, rather than choosing $\gamma_{Y}$, we may choose

$$
\omega_{Y}=\beta_{X, Y}^{o p} * \operatorname{Res}\left(\gamma_{X}\right) * \beta_{\operatorname{dec}(X, Y)}
$$

which satisfies $\operatorname{Res}\left(\gamma_{X}\right)$ is homotopic to $\omega_{Y}$. At the end of this section, we will produce a homotopy commutative diagram of spectra (see Lemma 5.8)

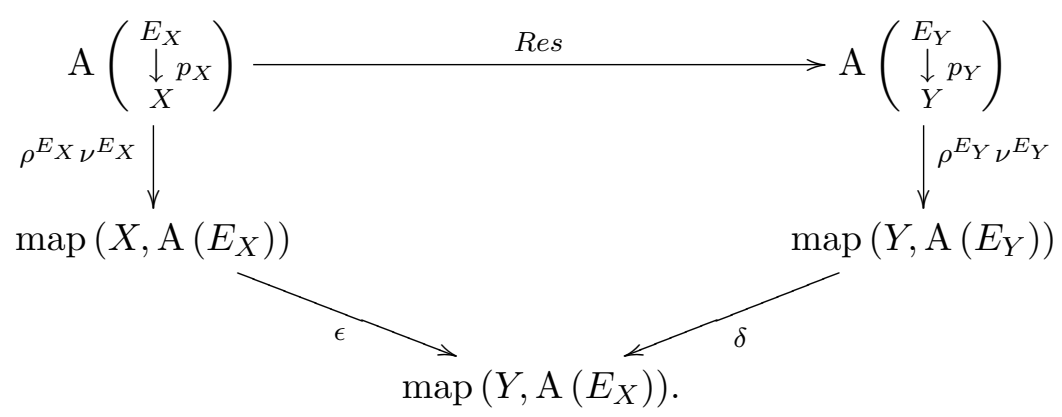

As a consequence, one may choose $H_{X}$ to be the image of $\gamma_{X}$ and $H_{Y}$ to be the image of $\omega_{Y}$ so $\epsilon H_{X} \simeq \delta H_{Y}$ implies the homotopies are appropriately compatible. Thereby one produces the required (induced) homotopy between the maps on homotopy cofibers, from $\tau_{\mathrm{A}}\left(p_{X, Y}\right) \Psi(\alpha)$ to $\tau_{\mathrm{A}}^{\text {dec }}\left(p_{X, Y}\right) \Psi(\alpha)$.

We now proceed with the verification of the strong additivity property for our candidate transfer $\mathbb{T}$, based upon the technical results which will fill the remainder of this section. 
Proposition 5.3. For a decomposed perfect fibration $p_{X}$ as above, there is a weak equivalence (of weak maps)

$$
\mathbb{T}(p) \sim \Sigma^{\infty}\left(k^{1}\right) \mathbb{T}\left(p_{1}\right)+\Sigma^{\infty}\left(k^{2}\right) \mathbb{T}\left(p_{2}\right)-\Sigma^{\infty}\left(k^{0}\right) \mathbb{T}\left(p_{0}\right),
$$

that is, the composites agree in the homotopy category of spectra.

Proof. This is a consequence of Proposition 5.6 and natural additivity of the assembly map (up to a natural homotopy in the category of spectra), which combine to say there is a weak equivalence (of weak maps)

$$
\tau_{\mathrm{A}}(p) \Psi(\alpha) \sim \mathrm{A}\left(k^{1}\right) \tau_{\mathrm{A}}\left(p_{1}\right) \Psi(\alpha)+\mathrm{A}\left(k^{2}\right) \tau_{\mathrm{A}}\left(p_{2}\right) \Psi(\alpha)-\mathrm{A}\left(k^{0}\right) \tau_{\mathrm{A}}\left(p_{0}\right) \Psi(\alpha) .
$$

Then we use the fact that the trace map is a natural additive map (again, up to a natural homotopy in the category of spectra) to see

$$
\begin{aligned}
& \Psi(\operatorname{tr}) \circ\left[\mathrm{A}\left(k^{1}\right) \tau_{\mathrm{A}}\left(p_{1}\right) \Psi(\alpha)+\mathrm{A}\left(k^{2}\right) \tau_{\mathrm{A}}\left(p_{2}\right) \Psi(\alpha)-\mathrm{A}\left(k^{0}\right) \tau_{\mathrm{A}}\left(p_{0}\right) \Psi(\alpha)\right] \sim \\
& \Sigma^{\infty}\left(k^{1}\right) \Psi(\operatorname{tr}) \tau_{\mathrm{A}}\left(p_{1}\right) \Psi(\alpha)+\Sigma^{\infty}\left(k^{2}\right) \Psi(\operatorname{tr}) \tau_{\mathrm{A}}\left(p_{2}\right) \Psi(\alpha)-\Sigma^{\infty}\left(k^{0}\right) \Psi(\operatorname{tr}) \tau_{\mathrm{A}}\left(p_{0}\right) \Psi(\alpha) .
\end{aligned}
$$

We next begin verifying the technical background by working toward the verification that the homotopy Euler characteristic is a lift of $p_{X}^{*} \alpha$. Recall the bivariant A-theory space of a fibration, and the parametrized Euler characteristic of Williams [36], discussed in subsection 2.3.

Definition 5.4. Let $\operatorname{simp} X$ denote the category (poset) of simplices of $X$ under inclusion. Then $\mathrm{A}_{X}\left(E_{X}\right)$ will denote the spectrum $\underset{\sigma \in \operatorname{simp} X}{\operatorname{hocolim}} \mathrm{A}\left(E_{X}^{\sigma}\right)$, where $E_{X}^{\sigma}$ is the restriction of $E_{X}$ over $\sigma \subset X$.

We will be carefully considering the composite map of spectra

$$
\begin{aligned}
& \mathrm{A}\left(\begin{array}{l}
E_{X} \\
\downarrow_{X} p_{X} \\
X
\end{array}\right) \stackrel{\nu^{E_{X}}}{\longrightarrow} \operatorname{holimp}_{\sigma \in \operatorname{simp} X} \mathrm{~A}\left(E_{X}^{\sigma}\right) \stackrel{\rho^{E_{X}}}{\longrightarrow} \operatorname{map}\left(X, \mathrm{~A}_{X}\left(E_{X}\right)\right) \\
& \downarrow\left(u^{E} X\right) * \\
& \operatorname{map}\left(X, \mathrm{~A}\left(E_{X}\right)\right) \text {. }
\end{aligned}
$$

Here $\nu^{E_{X}}$ is a Thomason homotopy inverse limit map (as in [36, page 12]). The map $\rho^{E_{X}}$ is defined, using the Bousfield-Kan [10] models for homotopy (co)limits, essentially by taking a natural transformation (on $\operatorname{simp} X)$ from $\Sigma^{\infty}\left(|\operatorname{simp} X / ?|_{+}\right)$ 
to $\mathrm{A}\left(E_{X}^{?}\right)$ and sending it to the induced map on hocolims and then exploiting the usual isomorphism of spectra

$$
\operatorname{Sp}\left(\Sigma^{\infty}\left(|\operatorname{simp} X|_{+}\right), \mathrm{A}_{X}\left(E_{X}\right)\right) \approx \operatorname{map}\left(X, \mathrm{~A}_{X}\left(E_{X}\right)\right) .
$$

Finally, $u^{E_{X}}: \mathrm{A}_{X}\left(E_{X}\right) \rightarrow \mathrm{A}\left(E_{X}\right)$ is the natural map constructed from the definitions.

With this definition, we also have a description of $\nu^{E_{X}}(\chi(p)) \in \Omega^{\infty} \operatorname{holim}_{\sigma \in \operatorname{sim} X} \mathrm{~A}\left(E_{X}^{\sigma}\right)$ as the class which in each $\Omega^{\infty} \mathrm{A}\left(E_{X}^{\sigma}\right)$ corresponds to the retractive space

$$
E_{X}^{\sigma} \sqcup E_{X}^{\sigma} \approx E_{X}^{\sigma} \times S^{0} \leftrightarrows E_{X}^{\sigma}
$$

A decomposed version can also be described fairly explicitly (see [13, page 256]). Furthermore, both of these definitions are extended to the relative case using Def. 2.2 .

Lemma 5.5. The image of $\chi\left(p_{X}\right)$ under $\Omega^{\infty}\left(u^{E_{X}}\right)_{*} \rho^{E_{X}} \nu^{E_{X}}$ is (the adjoint of) the composite of the assembly and the transfer

$$
\Sigma^{\infty}\left(X_{+}\right) \stackrel{\alpha}{\longrightarrow} \mathrm{A}(X) \stackrel{p_{X}^{*}}{\longrightarrow} \mathrm{A}\left(E_{X}\right) .
$$

Similarly, the image of $\chi\left(p_{\operatorname{dec}(X)}\right)$ under $\Omega^{\infty}\left(u^{E_{X}}\right)_{*} \rho^{E_{X}} \nu^{E_{X}}$ is the adjoint of the expected loop space sum

$$
\mathrm{A}\left(k^{1}\right) p_{1}^{*} \alpha+\mathrm{A}\left(k^{2}\right) p_{2}^{*} \alpha-\mathrm{A}\left(k^{0}\right) p_{0}^{*} \alpha
$$

of the composites of the assembly and transfer maps for the pieces of the decomposition (pushed into the total space).

Proof. We begin with establishing the following homotopy commutative diagram

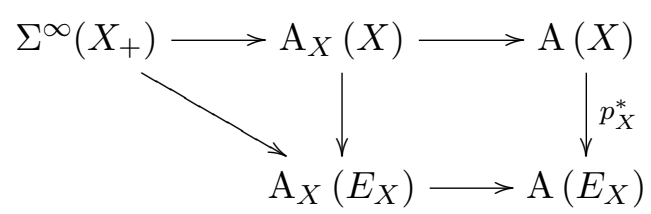

where the vertical maps are given by pullback over $p_{X}$ at the level of retractive spaces. The square commutes up to homotopy by naturality (up to isomorphism) of the pullback construction of retractive spaces. To see the triangle commutes 
up to homotopy, consider the following homotopy commutative diagram, where the vertical maps are induced by transfers over $p_{X}$

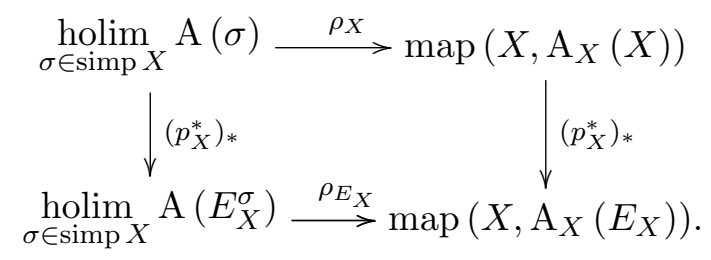

Here, the image of the $\nu^{X}(\chi(i d))$ under $\left(p_{X}^{*}\right)_{*} \rho_{X}$ is the upper composite in the triangle diagram. Similarly, the image of $\nu^{X}(\chi(i d))$ around the lower path through this diagram is (up to homotopy) the lower map in the triangle by definition, since the transfer of $\nu^{X}(\chi(i d))$ agrees with $\nu^{E_{X}}(\chi(p))$ by the explicit description of these classes given above.

Now the lower composite in the pentagon diagram represents the image of $\chi\left(p_{X}\right)$ under $\Omega^{\infty}\left(u^{E_{X}}\right)_{*} \rho^{E_{X}} \nu^{E_{X}}$ by construction. However, since the identity fibration is a smooth fiber bundle, Theorems 5.4 and 8.5 of Dwyer, Weiss, and Williams [17] along with naturality of (fiberwise) assembly imply the horizontal composite across the top of the pentagon diagram is homotopic to the assembly map. Since the right vertical in the pentagon diagram is the transfer of $p_{X}$, this completes the proof for that claim.

The claim for $\chi\left(p_{\operatorname{dec}(X)}\right)$ follows from above by looking at each $p_{i}$ separately, since the maps $\nu^{E_{X}}, \rho^{E_{X}}$, and $u^{E_{X}}$ are all natural in the variable $E_{X}$.

Let $H_{X}$ indicate the homotopy which is the image of $\gamma_{X}$ in map $\left(X, \mathrm{~A}\left(E_{X}\right)\right)$, and $H_{Y}$ similarly the image of $\omega_{Y}$. We will also use $l: E_{Y} \rightarrow E_{X}$ to indicate the inclusion on total spaces of the sub-fibration.

Proposition 5.6. Given a $C W$-pair $(X, Y)$ as above, there is a natural (in pairs) homotopy between the two homotopies $\left.\left(H_{X}\right)\right|_{Y}$ and $\mathrm{A}(l) \circ H_{Y}$ in the category of spectra. Hence there is a natural homotopy between $\tau_{\mathrm{A}}\left(p_{X, Y}\right) \Psi(\alpha)$ and $\tau_{\mathrm{A}}^{\operatorname{dec}}\left(p_{X, Y}\right) \Psi(\alpha)$.

Proof. By natural homotopy commutativity of the diagram given by Lemma 5.8 and the various lifts established in Lemma 5.5, it will suffice to see there is a natural homotopy in $\mathrm{A}\left(\begin{array}{c}E_{Y} \\ \downarrow \\ Y\end{array} p_{Y}\right)$ between $\operatorname{Res}\left(\gamma_{X}\right)$ and $\omega_{Y}$. However, this is 
a consequence of the construction of $\omega_{Y}$ from $\operatorname{Res}\left(\gamma_{X}\right)$ and two other natural paths.

The second claim follows from the first by employing Def. 2.2 and the compatibility of the homotopies established in the first claim.

We now have a pair of technical lemmas which will allow us to verify the natural homotopy commutativity of our key diagram. First we have a consequence of the Bousfield-Kan [10] models for homotopy limits and colimits.

Lemma 5.7. Suppose $F: \mathcal{C} \rightarrow \mathrm{Sp}$ and $\varphi: \mathcal{D} \rightarrow \mathcal{C}$ are functors, with $\mathcal{C}$ and $\mathcal{D}$ small categories. Then the following diagram commutes up to a natural homotopy

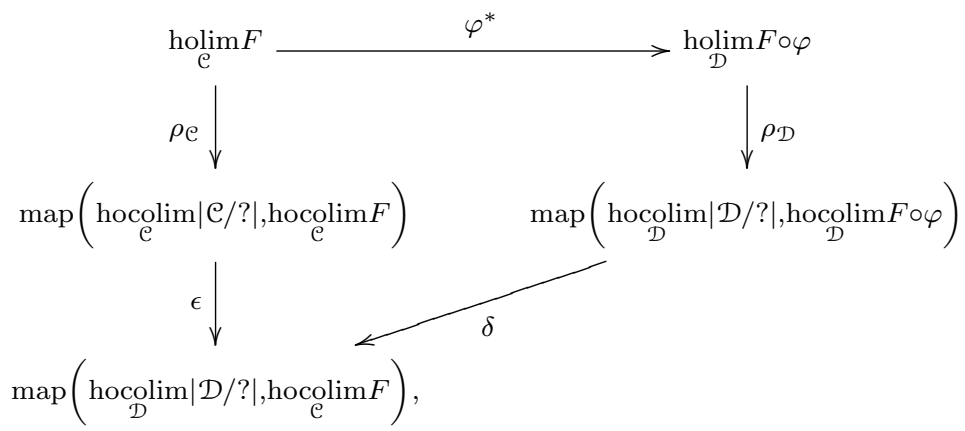

where $\epsilon$ denotes precomposition by

$\operatorname{hocolim} \varphi / ?: \underset{\mathcal{D}}{\operatorname{hocolim}}|\mathcal{D} / ?| \rightarrow \underset{\mathcal{C}}{\operatorname{hocolim}}|\mathcal{C} / ?|$

and $\delta$ denotes postcomposition by

$$
\underset{\mathcal{D}}{\operatorname{hocolim}}(F \circ \varphi) \rightarrow \underset{\mathfrak{C}}{\operatorname{hocolim} F}
$$


Lemma 5.8. The diagram

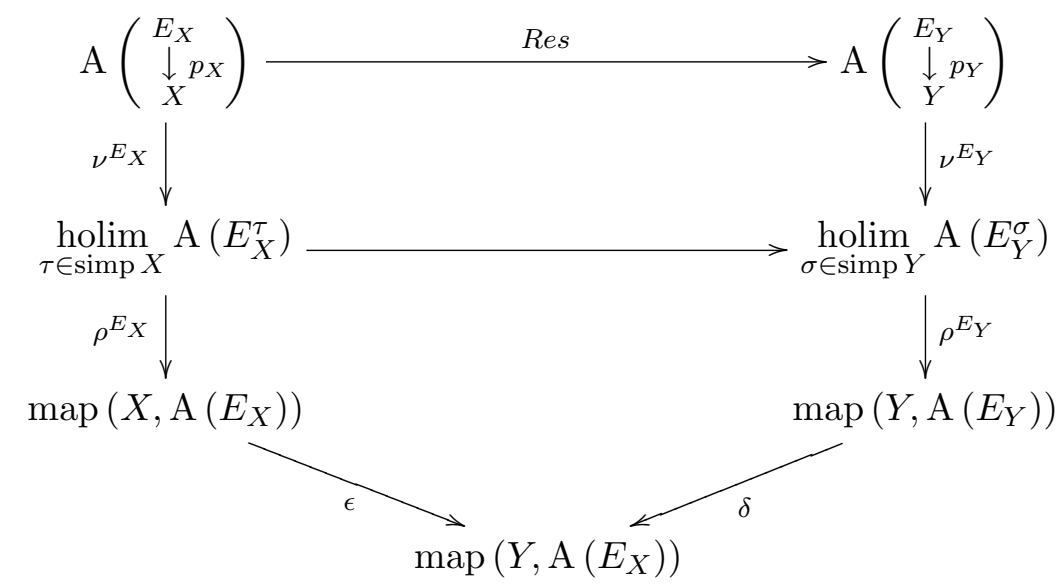

commutes up to a natural homotopy in the category of spectra.

Proof. The homotopy commutativity of the top square is just an observation about the naturality of the Thomason homotopy limit problem map

$$
\nu_{*}^{E_{X}}: \mathrm{A}\left(\begin{array}{c}
E_{X} \\
\downarrow \\
p_{X}
\end{array}\right) \rightarrow \underset{\tau \in \operatorname{simp} X}{\operatorname{holim}} \mathrm{A}\left(E_{X}^{\tau}\right),
$$

as discussed by Williams [36, page 12].

The lower pentagon is in part an application of Lemma 5.7, with $\mathcal{C}=\operatorname{simp} X$, $\mathcal{D}=\operatorname{simp} Y, \varphi$ the map induced by the topological inclusion $Y \rightarrow X$ and $F$ the value of $\mathrm{A}(?)$ at the pullback of $E_{X}$ over the inclusion of a given simplex, or explicitly $F(\sigma)=\mathrm{A}\left(E_{X}^{\sigma}\right)$. Notice if $\sigma=\varphi(\tau)$, we see $E_{X}^{\sigma} \approx E_{Y}^{\tau}$ by construction, hence

$$
(F \circ \varphi)(\tau)=\mathrm{A}\left(E_{X}^{\sigma}\right)=\mathrm{A}\left(E_{Y}^{\tau}\right) .
$$

Thus, we have the natural homotopy commutativity of

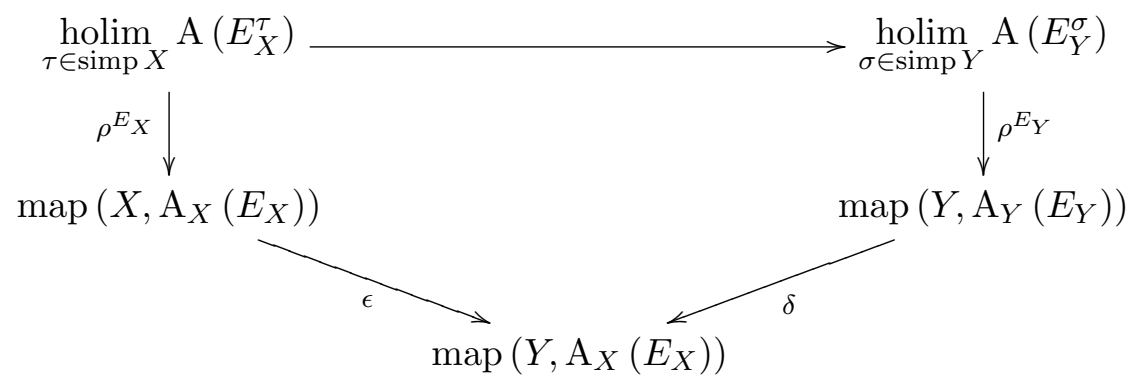


Now the following diagram commutes, where the vertical maps are induced by $u^{E_{X}}\left(\right.$ or $\left.u^{E_{Y}}\right)$

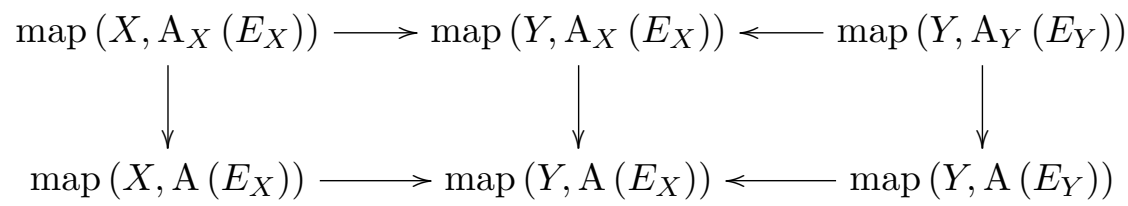

which completes the proof.

\section{REFERENCES}

[1] Douglas R. Anderson. The obstruction to the finiteness of the total space of a flat bundle. Amer. J. Math., 95:281-293, 1973.

[2] Bernard Badzioch and Wojciech Dorabiała. Additivity for parametrized topological Euler characteristic and Reidemeister torsion. K-theory, 38 (2007), no. 1, 1-22.

[3] Bernard Badzioch, Wojciech Dorabiała, and Bruce Williams. Smooth parametrized torsion - a manifold approach. Adv. Math., 221 (2009), no. 2, 660-680.

[4] J. C. Becker and D. H. Gottlieb. The transfer map and fiber bundles. Topology, 14:1-12, 1975.

[5] J. C. Becker and D. H. Gottlieb. Transfer maps for fibrations and duality. Compositio Math., 33(2):107-133, 1976.

[6] James C. Becker and Reinhard E. Schultz. Axioms for bundle transfers and traces. Math. Z., 227(4):583-605, 1998.

[7] M. Bökstedt, W. C. Hsiang, and I. Madsen. The cyclotomic trace and algebraic $K$-theory of spaces. Invent. Math., 111(3):465-539, 1993.

[8] Marcel Bökstedt. Topological hochschild homology. preprint Bielefeld, 1985.

[9] A. K. Bousfield and E. M. Friedlander. Homotopy theory of $\Gamma$-spaces, spectra, and bisimplicial sets. In Geometric applications of homotopy theory (Proc. Conf., Evanston, Ill., 1977), II, volume 658 of Lecture Notes in Math., pages 80-130. Springer, Berlin, 1978.

[10] A. K. Bousfield and D. M. Kan. Homotopy limits, completions and localizations. SpringerVerlag, Berlin, 1972. Lecture Notes in Mathematics, Vol. 304.

[11] Dan Burghelea. Automorphisms of manifolds. In Algebraic and geometric topology (Proc. Sympos. Pure Math., Stanford Univ., Stanford, Calif., 1976), Part 1, Proc. Sympos. Pure Math., XXXII, pages 347-371. Amer. Math. Soc., Providence, R.I., 1978.

[12] Jean Cerf. La stratification naturelle des espaces de fonctions différentiables réelles et le théorème de la pseudo-isotopie. Inst. Hautes Études Sci. Publ. Math., (39):5-173, 1970.

[13] Wojciech Dorabiała. The double coset theorem formula for algebraic $K$-theory of spaces. K-Theory, 25(3):251-276, 2002.

[14] Wojciech Dorabiała and Mark W. Johnson. The product theorem for parametrized homotopy Reidemeister torsion. J. Pure Appl. Algebra, 196(1):53-90, 2005. 
[15] Christopher L. Douglas. Trace and transfer maps in the algebraic $K$-theory of spaces. $K$ Theory, 36(1-2):59-82 (2006), 2005.

[16] Bjørn Ian Dundas, Thomas G. Goodwillie and Randy McCarthy. The local structure of algebraic K-theory, 9th June 2004, http://www.math.ntnu.no/ dundas/PP/040609b.pdf.

[17] W. Dwyer, M. Weiss, and B. Williams. A parametrized index theorem for the algebraic K-theory Euler class. Acta Math., 190(1):1-104, 2003.

[18] A. D. Elmendorf, I. Kriz, M. A. Mandell, and J. P. May. Rings, modules, and algebras in stable homotopy theory, volume 47 of Mathematical Surveys and Monographs. American Mathematical Society, Providence, RI, 1997. With an appendix by M. Cole.

[19] Thomas G. Goodwillie. Relative algebraic K-theory and cyclic homology. Ann. of Math. (2), 124(2):347-402, 1986.

[20] Mark Hovey, Brooke Shipley, and Jeff Smith. Symmetric spectra. J. Amer. Math. Soc., 13(1):149-208, 2000.

[21] Kiyoshi Igusa. The stability theorem for smooth pseudoisotopies. K-Theory, 2(1-2):vi+355, 1988.

[22] Daniel M. Kan. A combinatorial definition of homotopy groups. Ann. of Math. (2), 67:282312, 1958.

[23] John R. Klein and Bruce Williams. The refined transfer, bundle structures and algebraic K-theory, J. Topol. 2, 2009, no. 2, 321-345.

[24] Wolfgang Lück. The transfer maps induced in the algebraic $K_{0}$-and $K_{1}$-groups by a fibration. I. Math. Scand., 59(1):93-121, 1986.

[25] Wolfgang Lück. The transfer maps induced in the algebraic $K_{0^{-}}$and $K_{1}$-groups by a fibration. II. J. Pure Appl. Algebra, 45(2):143-169, 1987.

[26] Wolfgang Lück. Transformation groups and algebraic K-theory, volume 1408 of Lecture Notes in Mathematics. Springer-Verlag, Berlin, 1989. Mathematica Gottingensis.

[27] Ib Madsen. Algebraic $K$-theory and traces. In Current developments in mathematics, 1995 (Cambridge, MA), pages 191-321. Internat. Press, Cambridge, MA, 1994.

[28] Christian Schlichtkrull. The transfer map in topological Hochschild homology. J. Pure Appl. Algebra, 133(3):289-316, 1998.

[29] Arne Strøm. Note on cofibrations. II. Math. Scand., 22:130-142 (1969), 1968.

[30] Friedhelm Waldhausen. Algebraic $K$-theory of topological spaces. I. In Algebraic and geometric topology (Proc. Sympos. Pure Math., Stanford Univ., Stanford, Calif., 1976), Part 1, Proc. Sympos. Pure Math., XXXII, pages 35-60. Amer. Math. Soc., Providence, R.I., 1978.

[31] Friedhelm Waldhausen. Algebraic K-theory of topological spaces. II. In Algebraic topology, Aarhus 1978 (Proc. Sympos., Univ. Aarhus, Aarhus, 1978), volume 763 of Lecture Notes in Math., pages 356-394. Springer, Berlin, 1979.

[32] Friedhelm Waldhausen. Algebraic $K$-theory of spaces, a manifold approach. In Current trends in algebraic topology, Part 1 (London, Ont., 1981), volume 2 of CMS Conf. Proc., pages 141-184. Amer. Math. Soc., Providence, R.I., 1982. 
[33] Friedhelm Waldhausen. Algebraic K-theory of spaces. In Algebraic and geometric topology (New Brunswick, N.J., 1983), volume 1126 of Lecture Notes in Math., pages 318-419. Springer, Berlin, 1985.

[34] Friedhelm Waldhausen. On the construction of the Kan loop group. Doc. Math., 1:No. 05, 121-126 (electronic), 1996.

[35] Michael Weiss and Bruce Williams. Assembly. In Novikov conjectures, index theorems and rigidity, Vol. 2 (Oberwolfach, 1993), volume 227 of London Math. Soc. Lecture Note Ser., pages 332-352. Cambridge Univ. Press, Cambridge, 1995.

[36] Bruce Williams. Bivariant Riemann Roch theorems. In Geometry and topology: Aarhus (1998), volume 258 of Contemp. Math., pages 377-393. Amer. Math. Soc., Providence, RI, 2000 .

Wojciech Dorabiała

Department of Mathematics

Penn State Altoona

Altoona, PA 16601-3760

E-mail: wud2@psu.edu

Mark W. Johnson

Department of Mathematics

Penn State Altoona

Altoona, PA 16601-3760

E-mail: mwj3@psu.edu 